\title{
Dynamical reflection equation*
}

\author{
P. P. Kulish ${ }^{\dagger}$ and A. I. Mudrov
}

To the memory of Joseph Donin

\begin{abstract}
${ }^{\dagger}$ St.-Petersburg Department of Steklov Mathematical Institute, Fontanka 27, 191011 St.-Petersburg, Russia, e-mail: kulish@pdmi.ras.ru

$\ddagger$ Department of Mathematics, Bar Ilan University, 52900 Ramat Gan, Israel, e-mail: mudrova@math.biu.ac.il

${ }^{\natural}$ Max-Planck Institut für Mathematik, Vivatsgasse 7, D-53111 Bonn, Germany. e-mail: mudrov@mpim-bonn.mpg.de
\end{abstract}

\begin{abstract}
We construct a dynamical reflection equation algebra, $\tilde{\mathcal{K}}$, via a dynamical twist of the ordinary reflection equation algebra. A dynamical version of the reflection equation is deduced as a corollary. We show that $\tilde{\mathcal{K}}$ is a right comodule algebra over a dynamical analog of the Faddeev-Reshetikhin-Takhtajan algebra equipped with a structure of right bialgebroid. We introduce dynamical trace and use it for constructing central elements of $\tilde{\mathcal{K}}$.
\end{abstract}

Key words: Dynamical Yang-Baxter equation, dynamical reflection equation, quantum groupoids. AMS classification codes: 17B37, 81R50.

\section{Contents}

1 Introduction

${ }^{*}$ This research is supported in part by the Israel Academy of Sciences grant no. 8007/99-03, the Emmy Noether Research Institute for Mathematics, the Minerva Foundation of Germany, the Excellency Center "Group Theoretic Methods in the study of Algebraic Varieties" of the Israel Science foundation, and by the RFBR grant no. 03-01-00593. 
2 Bialgebroids $\quad 5$

2.1 Left bialgebroids . . . . . . . . . . . . . . . . . . 5

2.2 Right bialgebroids . . . . . . . . . . . . . . . . . . . 6

2.3 Some examples . . . . . . . . . . . . . . . . . . . . . . . . 7

3 Dynamical FRT and RE algebras $\quad 9$

3.1 dFRT algebra associated with a dvnamical matrix $\tilde{S} \ldots \ldots$

3.2 Extended dFRT algebra . . . . . . . . . . . . . . . . . . . . . 12

3.3 Categorv of comodules over a bialgebroid . . . . . . . . . . . . . . 13

3.4 dRE algebra associated with a dvnamical matrix $\tilde{S}$. . . . . . . . . . . 14

4 Twisting the FRT and RE algebras 16

4.1 Twist and twisted tensor square . . . . . . . . . . . . . . . 17

4.2 How a twist of the Hopf algebra affects the RE algebra . . . . . . . . . . . . 18

5 Algebra of symmetric dynamical tensors $\quad 19$

5.1 Some definitions and notation . . . . . . . . . . . . . . . 19

5.2 Dvnamical twist and R-matrix . . . . . . . . . . . . . . . . 21

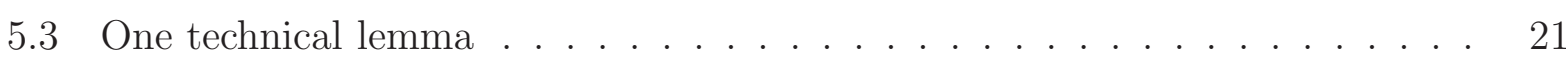

5.4 An anti-automorphism of $\check{U}^{n} \ldots \ldots \ldots \ldots$

6 A gauge transformation of dvnamical twist $\quad 24$

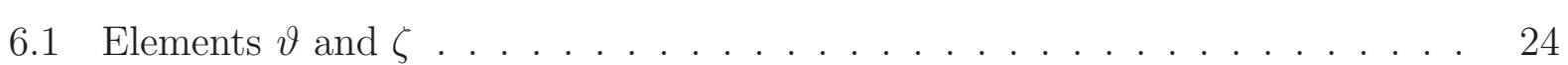

6.2 The gauge transformation . . . . . . . . . . . . . . . . . . . 25

6.3 Svmmetries of dvnamical R-matrix . . . . . . . . . . . . . . . 26

7 Dynamical twist of the RE algebra $\quad 27$

7.1 Dvnamical twist and quantum groupoids . . . . . . . . . . . . . . . . 27

7.2 dFRT algebra via dvnamical twist . . . . . . . . . . . . . . . . . . . . 29

$7.3 \mathrm{dRE}$ algebra via dvnamical twist . . . . . . . . . . . . . . 31

7.4 A homomorphism of the $\mathrm{dRE}$ algebra to $\mathcal{U} \otimes \mathcal{L} \ldots \ldots . \ldots 33$

8 Dvnamical trace and center of the dRE algebra $\quad 34$

8.1 Dvnamical trace. . . . . . . . . . . . . . . . . . . . . 34

8.2 On the center of the dRE algebra . . . . . . . . . . . . 36 


\section{Introduction}

The dynamical Yang-Baxter equation (dYBE) appeared in integrable models of conformal field theories $[\mathrm{AF}, \mathrm{ABB}, \mathrm{Fad}, \mathrm{F}, \mathrm{GN}]$. It is a generalization of the ordinary Yang-Baxter equation (YBE), which is the most important equation of the quantum inverse scattering method. In the simplest version of abelian base, the dYBE and related structures were studied in [EV1, EV2, ES1, S], see also the lecture course [ES1] and references therein. The general formulation of dYBE (over arbitrary base) was motivated by its relation to equivariant quantization on G-spaces and developed in [DM1] that formulation was based on ideas of EV1, EV2, $\mathrm{Xu2}$.

The theory of the YBE may be considered as a part of the Hopf algebra (more generally, bialgebra) theory, Dr1. The natural analog of Hopf algebra for the dYBE is bialgebroid. The remarkable distinction of bialgebroids from Hopf algebras is replacement of the field of scalars by an algebra that is non-commutative in general; it is called "base algebra". Roughly speaking, the connection between the theory of dYBE and bialgebroids is established via the base algebra, whose spectrum is set to be the space of dynamical parameter.

Many standard concepts of the Hopf algebra theory, such as tensor product of modules, twists, module and comodule algebras can be formulated for bialgebroids, Szzl, Xu1, Sch]. One can also define a quasitriangular structure on bialgebroids, which in special cases is expressed through a dynamical R-matrix, DM2. In the present paper, we study dynamical analogs of two important objects of the Hopf algebra theory: the Faddeev-ReshetikhinTakhtajan (FRT) and reflection equation (RE) algebras, FRT, KSkl, KS]. The latter is given our special consideration.

A naive point of view on the $\mathrm{dRE}$ is to take for it the equation satisfied by the squared permutation in the dynamical category, DM1, as it goes in the Hopf algebra theory. The question is what are the properties of that equation and what algebraic structures are behind it. The presented analysis of those structures supports that "naive" point of view and shows that the theory of the dRE is analogous to the theory of its non-dynamical counterpart.

Below we obtain the following results. We develop a theory of dynamical reflection equation over arbitrary base (by a base we understand a pair of a Hopf algebra and its base algebra, see [DM1]). In particular, we associates to any matrix solution $\tilde{R}$ the dYBE over a base algebra $\mathcal{L}$ a right $\mathcal{L}$-bialgebroid called dFRT algebra. We define a dRE algebra as a right comodule algebra over the dFRT bialgebroid. We concentrate our study on the situation

when $\tilde{R}$ is the image of the universal dynamical R-matrix $\tilde{\mathcal{R}}$ obtained by a dynamical twist 
from a constant universal R-matrix, $\tilde{\mathcal{R}}=\mathcal{F}_{21}^{-1} \mathcal{R} \mathcal{F}$. Under this assumption, we prove that the dFRT and dRE algebras are modules over certain quantum groupoids and can be obtained by dynamical twists from the FRT and RE algebras. For twisted dynamical R-matrices, we introduce dynamical trace and prove that traces of powers of the dynamical RE matrices lie in the center of the dRE algebra.

Let us mention two papers that are relevant to the subject in question. A version of quadratic algebra depending on a dynamical parameter corresponding to the abelian base was proposed in $\mathrm{NAR}$. That algebra is different from what is studied in the present paper. It is defined by four different matrices, whose precise meaning has to be understood (except for one, which is a solution of the dYBE). Another version of dynamical quadratic algebra was suggested in [FHS. Essentially, that is a particular case of our dRE algebra specialized for the abelian base.

The article is organized in the following way.

- Section 2 recalls the definition of left and right bialgebroids and gives some examples.

- Section 3 introduces dFRT and dRE algebras for any dynamical permutation $\tilde{S}$.

- Section 4 discusses a way of constructing the dRE algebra by dynamical twist, following an analogy with the Hopf algebra case.

- Section 5 develops a technical machinery necessary for realization of the strategy adopted in Section 4 .

- Section [ 6 studies symmetries of dynamical twists and dynamical R-matrices.

- Section 7 constructs the dFRT and dRE algebras via dynamical twists of the ordinary FRT and RE algebras.

- Section 8 introduces dynamical trace used for constructing central elements of the dRE algebra (in the twisted case).

\section{Notation and conventions}

All the vector spaces are over the ground field $k$ of zero characteristic. Algebras are associative unital algebras over the ground field. The subscript op denotes the opposite multiplication. To make formulas more readable, we denote the inverse by bar, $\bar{a}:=a^{-1}$. Coproduct, counit and antipode are denoted by $\Delta$, $\varepsilon$, and $\gamma$ without reference to a particular Hopf algebra or bialgebroid if they are clear from the context. The symbol $\otimes$ stands for the tensor 
product over the ground field. Tensors products over other rings are indicated explicitly. We use the standard Sweedler notation for the coproduct $\Delta(x)=x^{(1)} \otimes x^{(2)}$. Similar convention is used for a Hopf algebra coaction, say, $\delta(v)=v^{(1)} \otimes v^{[\infty]}$, where the factor of the same nature as $v$ is marked with [ $\infty$ ] for left- and with [0] for right comodules. The iterated coproducts are denoted by $\Delta^{2}:=\Delta, \Delta^{3}:=(\Delta \otimes \mathrm{id}) \circ \Delta$ and so on. Similar notation is used for iterated coactions. Factors of tensor objects are labelled by positive integers in the standard way. Notation $F_{(12) 3}$ or $F_{1(234)}$ etc means $(\Delta \otimes \mathrm{id})(F)$ or $\left(\mathrm{id} \otimes \Delta^{3}\right)(F)$ etc for an element $F$ from the tensor square of a Hopf algebra. More special notation will be introduced in Subsection 5.1 .

\section{Bialgebroids}

The concept of bialgebroid generalizes that of bialgebra. Bialgebroids naturally arise in the theory of dYBE as bialgebras (Hopf algebras) in the theory of YBE. Similarly to bialgebras, representations of a bialgebroid form a monoidal category. The distinction from the bialgebra case is that such monoidal category has no fiber functor to the vector category. Rather, it has a fiber functor to the monoidal category of $\mathcal{L}$-bimodules, where $\mathcal{L}$ is an algebra called base.

Below we give, for further convenience, definitions of left and right bialgebroids. For details, the reader is referred to $[\mathrm{Lu}]$ and $[\mathrm{Szl}]$. Bialgebroids in connection with the dYBE were studied in [Xu1] and DM2].

Suppose we are given with a pair of associative algebras $(\mathcal{L}, \mathcal{B})$, a homomorphism $s: \mathcal{L} \rightarrow$ $\mathcal{B}$ and anti-homomorphism $t: \mathcal{L} \rightarrow \mathcal{B}$ called source and target maps, respectively. Assume that their images commute in $\mathcal{B}$. One can consider the following two $\mathcal{L}$-bimodule structures on $\mathcal{B}$ induced by these maps. One of them is induced by the left regular representation, $\lambda . b . \mu:=s(\lambda) t(\mu) b, \lambda, \mu \in \mathcal{L}, b \in \mathcal{B}$. The other comes from the right regular action, $\lambda . b . \mu:=$ $b t(\lambda) s(\mu), \lambda, \mu \in \mathcal{L}, b \in \mathcal{B}$.

\section{$2.1 \quad$ Left bialgebroids}

In this subsection we fix the $\mathcal{L}$-bimodule structure on $\mathcal{B}$ defined via the left regular action. The tensor square $\mathcal{B} \otimes_{\mathcal{L}} \mathcal{B}$ is not an algebra, in general. However, it contains a natural algebra as a subspace. Namely, put

$$
\mathcal{B} \times_{\mathcal{L}} \mathcal{B}=\left\{z \in \mathcal{B} \otimes_{\mathcal{L}} \mathcal{B} \mid z(t(\lambda) \otimes 1)=z(1 \otimes s(\lambda)), \forall \lambda \in \mathcal{L}\right\}
$$


Clearly, $\mathcal{B} \times_{\mathcal{L}} \mathcal{B}$ is a unital associative algebra.

Definition 2.1. The quadruple $(\mathcal{B}, \mathcal{L}, s, t)$ is called a left bialgebroid over base $\mathcal{L}$ if there exist

1. a coassociative $\mathcal{L}$-bimodule map (comultiplication) $\Delta: \mathcal{B} \rightarrow \mathcal{B} \times_{\mathcal{L}} \mathcal{B}$ that is a homomorphisms of associative algebras.

2. a bimodule map (counit) $\varepsilon: \mathcal{B} \rightarrow \mathcal{L}$ such that $\varepsilon\left(1_{\mathcal{B}}\right)=1_{\mathcal{L}}$

$$
\begin{gathered}
\varepsilon(a(s \circ \varepsilon)(b))=\varepsilon(a b)=\varepsilon(a(t \circ \varepsilon)(b)), \quad a, b \in \mathcal{B}, \quad \text { and } \\
\left(\varepsilon \otimes_{\mathcal{L}} \operatorname{id}_{\mathcal{B}}\right) \circ \Delta=\operatorname{id}_{\mathcal{B}}=\left(\operatorname{id}_{\mathcal{B}} \otimes_{\mathcal{L}} \varepsilon\right) \circ \Delta
\end{gathered}
$$

under the identification $\mathcal{L} \otimes_{\mathcal{L}} \mathcal{B} \simeq \mathcal{B} \simeq \mathcal{B} \otimes_{\mathcal{L}} \mathcal{L}$.

Condition 2 implies the identities $\varepsilon \circ s=\varepsilon \circ t=\operatorname{id}_{\mathcal{L}}$ and makes $\mathcal{L}$ a left $\mathcal{B}$-module by

$$
a \vdash \lambda:=\varepsilon(a s(\lambda))=\varepsilon(a t(\lambda)), \quad a \in \mathcal{B}, \lambda \in \mathcal{L},
$$

where the right equality is a consequence of (2). The $\mathcal{L}$-bimodule structure on $\mathcal{L}$ induced by this action coincides with the standard one. The action (4) is called (left) anchor. In fact, the anchor determines the counit together with condition (2) by $\varepsilon(a)=a \vdash 1_{\mathcal{L}}$ for $a \in \mathcal{B}$.

Any left $\mathcal{B}$-module $V$ is a natural $\mathcal{L}$-bimodule. This correspondence is called the forgetful functor. Given two $\mathcal{B}$-modules $V$ and $W$, the tensor product $V \otimes_{\mathcal{L}} W$ acquires a left $\mathcal{B}$-module structure via the coproduct, due to condition 1 of Definition 2.1. The whole set of axioms from Definition 2.1 ensures that the left $\mathcal{B}$-modules form a monoidal category, with $\mathcal{L}$ being the unit object. The forgetful functor to the category of $\mathcal{L}$-bimodules is strong monoidal, i.e. preserves tensor products. Conversely, suppose there is a monoidal structure on the category of left $\mathcal{B}$-modules. Suppose the forgetful functor to the category of $\mathcal{L}$-bimodules is strong monoidal. Then $\mathcal{B}$ is a left $\mathcal{L}$-bialgebroid, see [Szl].

\subsection{Right bialgebroids}

The theory of right bialgebroids is fully parallel to the theory of left ones. Let us write out the definition for future convenience.

Now assume the alternative $\mathcal{L}$-bimodule structure on $\mathcal{B}$, that is specified by the right regular action. The algebra $\mathcal{B} \times_{\mathcal{L}} \mathcal{B}$ is defined in this case as

$$
\mathcal{B} \times_{\mathcal{L}} \mathcal{B}=\left\{z \in \mathcal{B} \otimes_{\mathcal{L}} \mathcal{B} \mid(s(\lambda) \otimes 1) z=(1 \otimes t(\lambda)) z, \forall \lambda \in \mathcal{L}\right\}
$$


Definition 2.2. The quadruple $(\mathcal{B}, \mathcal{L}, s, t)$ is called a right bialgebroid over base $\mathcal{L}$ if there exist

1. a coassociative $\mathcal{L}$-bimodule map homomorphism (comultiplication) $\Delta: \mathcal{B} \rightarrow \mathcal{B} \times_{\mathcal{L}} \mathcal{B}$ that is a homomorphisms of associative algebras.

2. a bimodule map (counit) $\varepsilon: \mathcal{B} \rightarrow \mathcal{L}$ such that $\varepsilon\left(1_{\mathcal{B}}\right)=1_{\mathcal{L}}$,

$$
\begin{gathered}
\varepsilon((s \circ \varepsilon)(a) b)=\varepsilon(a b)=\varepsilon((t \circ \varepsilon)(a) b), \quad a, b \in \mathcal{B}, \quad \text { and } \\
\left(\varepsilon \otimes_{\mathcal{L}} \operatorname{id}_{\mathcal{B}}\right) \circ \Delta=\operatorname{id}_{\mathcal{B}}=\left(\operatorname{id}_{\mathcal{B}} \otimes_{\mathcal{L}} \varepsilon\right) \circ \Delta
\end{gathered}
$$

under the identification $\mathcal{L} \otimes_{\mathcal{L}} \mathcal{B} \simeq \mathcal{B} \simeq \mathcal{B} \otimes_{\mathcal{L}} \mathcal{L}$.

Similarly to the left bialgebroids, one can define the (right) anchor action setting

$$
\lambda \dashv a:=\varepsilon(s(\lambda) a)=\varepsilon(t(\lambda) a), \quad a \in \mathcal{B}, \lambda \in \mathcal{L} .
$$

The anchor determines the counit by $\varepsilon(a)=1 \dashv a$ for $a \in \mathcal{B}$.

The right modules over right bialgebroids form a monoidal category with the fiber functor to the monoidal category of $\mathcal{L}$-bimodules.

Remark 2.3. It is possible to define twist and quasitriangular structure for bialgebroids, as well as for Hopf algebras. The reader is referred to [Xu1] and [DM2] for details.

\subsection{Some examples}

Let us give some examples of bialgebroids that arise in the theory of dYBE. More examples will appear bellow. Bialgebroids related to the dYBE are defined over a base that is commutative with respect to some permutation. More specifically, let us fix a Hopf algebra $\mathcal{H}$ with the coproduct $\Delta$, and invertible antipode $\gamma$. Recall from [DM1 that an $\mathcal{H}$-base algebra $\mathcal{L}$ is a left $\mathcal{H}$-module algebra and left $\mathcal{H}$-comodule algebra; it is a Yetter-Drinfeld module with respect to these structures and "commutative" in the following sense:

$$
\lambda \mu=\left(\lambda^{(1)} \triangleright \mu\right) \lambda^{[\infty]}, \quad \lambda, \mu \in \mathcal{L} .
$$

Here $\lambda \mapsto \lambda^{(1)} \otimes \lambda^{[\infty]} \in \mathcal{H} \otimes \mathcal{L}$ denotes the coaction $\delta$ and $\triangleright$ stands for the $\mathcal{H}$-action on $\mathcal{L}$. Algebraically, $\mathcal{L}$ can be defined as a module algebra over the double $\mathfrak{D H}=\mathcal{H} \bowtie \mathcal{H}_{\text {op }}^{*}$, Dr1], that is commutative with respect to the standard quasitriangular structure $\Theta \in \mathcal{H}_{o p}^{*} \otimes \mathcal{H} \subset$ 
$(\mathfrak{D H})^{\otimes 2}$. The coaction may be written in terms of $\Theta$ and the $\mathfrak{D H}$-action (for this we reserve notation $\triangleright$ throughout the whole paper)

$$
\delta: \lambda \mapsto \Theta_{2} \otimes \Theta_{1} \triangleright \lambda .
$$

Note that being a Yetter-Drinfeld module over $\mathcal{H}$ is immediate from this representation and the intertwining axiom for R-matrices. It is convenient to use representation (10), although all the results would be true without this simplifying assumption.

Example 2.4. Consider the smash product $\mathcal{L} \rtimes \mathfrak{D H}$ algebra with the multiplication

$$
(\lambda \otimes f)(\mu \otimes g):=\lambda\left(f^{(1)} \triangleright \mu\right) \otimes f^{(2)} g, \quad \lambda, \mu \in \mathcal{L}, \quad f, g \in \mathcal{H} .
$$

The algebra $\mathcal{L} \rtimes \mathfrak{D H}$ is a left $\mathcal{L}$-bialgebroid with the subalgebra $\mathcal{L} \rtimes \mathcal{H}$ being a sub-bialgebroid. The construction is due to $[\mathrm{Lu}$ and goes as follows. The source and target maps are set to be

$$
s(\lambda)=\lambda \otimes 1, \quad t(\lambda)=\bar{\Theta}_{1} \triangleright \lambda \otimes \bar{\Theta}_{2}
$$

The counit and coaction on $\mathcal{L} \rtimes \mathfrak{D H}$ are defined as

$$
\begin{aligned}
\varepsilon(\lambda \otimes h) & =\lambda \varepsilon_{\mathcal{H}}(h), \\
\Delta(\lambda \otimes h) & =\left(\lambda \otimes h^{(1)}\right) \otimes_{\mathcal{L}}\left(1 \otimes h^{(2)}\right) .
\end{aligned}
$$

The anchor action is given by $(\lambda \otimes h) \vdash \mu=\lambda(h \triangleright \mu)$, for $\lambda \otimes h \in \mathcal{L} \rtimes \mathfrak{D H}$ and $\mu \in \mathcal{L}$.

Example 2.5. Let $\mathcal{L}$ be an $\mathcal{H}$-base algebra with the $\mathcal{H}$-action $\triangleright$ and the coaction $\delta$. Then $\mathcal{L}_{o p}$ is a base algebra over $\mathcal{H}_{o p}$ with respect to the $\mathcal{H}_{o p}$-action $x \otimes \ell \mapsto \gamma^{-1}(x) \triangleright \ell$ and the same coaction $\delta$ considered now as a map $\mathcal{L}_{o p} \rightarrow \mathcal{H}_{o p} \otimes \mathcal{L}_{o p}$. Along the line of Example 2.4 one can construct the left $\mathcal{L}_{o p}$-bialgebroids $\mathcal{L}_{o p} \rtimes \mathfrak{D H} \mathcal{H}_{o p}$ and $\mathcal{L}_{o p} \rtimes \mathcal{H}_{o p}$.

Similarly to Hopf algebras, one can define biideals in bialgebroids. Namely, an $\mathcal{L}$ bimodule $\mathcal{J} \subset \mathcal{B}$ is called biideal if $\Delta(\mathcal{J}) \subset \mathcal{J} \otimes_{\mathcal{L}} \mathcal{B}+\mathcal{B} \otimes_{\mathcal{L}} \mathcal{J}$ and $\varepsilon(\mathcal{J})=0$. The latter condition holds if and only if $\mathcal{J}$ lies in the kernel of the anchor. The quotient of a bialgebroid by a biideal is a bialgebroid. Conversely, the kernel of a bialgebroid homomorphism is a biideal.

Example 2.6. The bialgebroid $\mathcal{L} \rtimes \mathfrak{D H}$ from 2.4 is not quasitriangular. Here is an example of quasitriangular bialgebroid (quantum groupoid) from [DM2]. The quotient $\mathfrak{D H}_{\mathcal{L}}$ of $\mathcal{L} \rtimes \mathfrak{D H}$ by the two-sided ideal generated by $\bar{\Theta}_{1} \triangleright \lambda \otimes \bar{\Theta}_{2}-\Theta_{2} \triangleright \lambda \otimes \Theta_{1}, \forall \lambda \in \mathcal{L}$, is again a left $\mathcal{L}$-bialgebroid (recall that bar means the inverse). It is quasitriangular, with the universal R-matrix obtained by a natural projection from the universal R-matrix of $\mathfrak{D H}$, see [DM2] for details. 


\section{Dynamical FRT and RE algebras}

Let $V$ be a finite dimensional $\mathcal{H}$-module and $\rho$ denote the homomorphism from $\mathcal{H}$ to $\operatorname{End}(V)$. Let $\left\{e_{j}^{i}\right\} \subset \operatorname{End}(V)$ denote the standard matrix base with the multiplication to $e_{j}^{i} e_{k}^{l}=\delta_{j}^{l} e_{k}^{i}$; here $\delta_{j}^{l}$ is the Kronecker symbol.

Let $\tilde{S}$ be an element from $\operatorname{End}^{\otimes 2}(V) \otimes \mathcal{L}$ that is invariant in the following sense:

$$
h \triangleright \tilde{S}=\left(\rho^{\otimes 2} \circ \Delta\right)\left(\gamma h^{(1)}\right) \tilde{S}\left(\rho^{\otimes 2} \circ \Delta\right)\left(h^{(2)}\right) \quad \text { for all } h \in \mathcal{H} .
$$

It follows from this transformation law that $\tilde{S}$ commutes with $\left(\rho^{\otimes 2} \circ \delta^{2}\right)(\lambda)$ for all $\lambda \in \mathcal{L}$ (here $\delta^{2}$ is the two-folded coaction). Put $\tilde{S}_{o p}:=\tilde{S}_{1} \rho\left(\gamma \Theta_{2}^{(2)}\right) \otimes \tilde{S}_{2} \rho\left(\gamma \Theta_{2}^{(1)}\right) \otimes \Theta_{1} \triangleright \tilde{S}_{\mathcal{L}}$.

In the next two subsections we associate with the matrix $\tilde{S}$ the dynamical analogs of two important algebras in the theory of quantum groups, the FRT and RE algebras.

\section{1 dFRT algebra associated with a dynamical matrix $\tilde{S}$}

We call an algebra $\mathcal{A}$ in the monoidal category of $\mathcal{L}$-bimodules an $\mathcal{L}$-bimodule algebra. It is an associative algebra whose multiplication and $\mathcal{L}$-bimodule structure are compatible, namely $(a . \lambda) b=a(\lambda . b)$ for all $a, b \in \mathcal{A}$ and $\lambda \in \mathcal{L}$. A natural example of an $\mathcal{L}$-bimodule algebra is obtained when the bimodule structure is induced by a homomorphism $\mathcal{L} \rightarrow \mathcal{A}$. This is the case if and only if the left and right $\mathcal{L}$-actions satisfy $\lambda .1_{\mathcal{A}}=1_{\mathcal{A}} \cdot \lambda$ for all $\lambda \in \mathcal{L}$.

Let $\left\{T_{j}^{i}\right\} \subset \operatorname{End}^{*}(V)$ be the dual base to $\left\{e_{i}^{j}\right\}$. Consider the space $\operatorname{End}^{*}(V)$ as a left $\mathcal{H}_{o p} \otimes \mathcal{H}$-module with respect to the action

$$
x \triangleright T:=T \rho(x), \quad y \triangleright T:=\rho(y) T,
$$

where $x \in \mathcal{H}, y \in \mathcal{H}_{o p}$, and $T:=\sum_{i, j} e_{i}^{j} \otimes T_{j}^{i}$. The action of $\mathcal{H}_{o p} \otimes \mathcal{H}$ naturally extends to an action on the free algebra $k\left\langle T_{j}^{i}\right\rangle:=k\left\langle\left\{T_{j}^{i}\right\}\right\rangle$ making the latter an $\mathcal{H}$-bimodule algebra. Consider the smash product $\mathfrak{F}(T):=k\left\langle T_{j}^{i}\right\rangle \rtimes\left(\mathcal{L}_{o p} \otimes \mathcal{L}\right)$ determined by the permutation relations

$$
\lambda T=T \rho\left(\lambda^{(1)}\right) \lambda^{[\infty]}, \quad \mu T=\rho\left(\mu^{(1)}\right) T \mu^{[\infty]}
$$

for $\lambda \in \mathcal{L}$ and $\mu \in \mathcal{L}_{\text {op }}$. It is easy to see that $\mathfrak{F}(T)$ is a left module algebra over the Hopf algebra $\left(\mathcal{H}_{o p}{ }^{\otimes} \mathfrak{D H}_{o p}\right) \otimes(\mathcal{H} \stackrel{\ominus}{\otimes} \mathfrak{D H})$, where $\mathfrak{D H}$ and $\mathfrak{D} \mathcal{H}_{o p}$ act essentially on the $\mathcal{L}$ and $\mathcal{L}_{o p}$ factors, respectively. Here $\stackrel{\mathcal{F}}{\otimes}$ denotes the twisted tensor product of two Hopf algebras by a bicharacter $\mathcal{F}$, see $[\mathrm{RS}]$ and also $[\mathrm{DM} 3$. We also regard $\mathfrak{F}(T)$ as an $\mathcal{H}$-bimodule by the Hopf algebra embedding

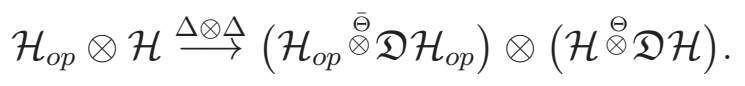


Consider the ideal $\mathcal{J}_{d F R T}$ in $\mathfrak{F}(T)$ generated by the relations

$$
\tilde{S}_{o p} T_{1} T_{2}=T_{1} T_{2} \tilde{S}
$$

where the matrix coefficients of $\tilde{S}_{o p}$ and $\tilde{S}$ belong respectively to $\mathcal{L}_{o p}$ and $\mathcal{L}$.

Definition 3.1. The dFRT algebra $\tilde{\mathcal{T}}$ associated with the matrix $\tilde{S}$ is the quotient of $\mathfrak{F}(T)$ by the ideal $\mathcal{J}_{d F R T}$.

It is straightforward to check, using (14), that $\mathcal{J}_{d F R T}$ (and therefore $\tilde{\mathcal{T}}$ ) is an $\mathcal{H}$-bimodule. The natural embedding

$$
(t \otimes s): \mathcal{L} \otimes \mathcal{L} \rightarrow k\left\langle T_{j}^{i}\right\rangle \rtimes\left(\mathcal{L}_{o p} \otimes \mathcal{L}\right)
$$

makes $\mathfrak{F}(T)$, and therefore $\tilde{T}$, an $\mathcal{L}$-bimodule via the right regular action. These $\mathcal{H}$ - and $\mathcal{L}$-bimodule structures on $\mathfrak{F}(T)$ and $\tilde{\mathcal{T}}$ amount to a structure of left module over the $\mathcal{L}_{o p} \otimes \mathcal{L}$ bialgebroid $\left(\mathcal{L}_{o p} \rtimes \mathcal{H}_{o p}\right) \otimes(\mathcal{L} \rtimes \mathcal{H})$; the latter is a tensor product of $\mathcal{L}_{o p^{-}}$and $\mathcal{L}$-bialgebroids, see Examples 2.4 and 2.5 .

Our next objective is to introduce a structure of right bialgebroid on $\tilde{\mathcal{T}}$. Let us start with the anchor action and the counit.

Lemma 3.2. The formulas

$$
\lambda \dashv \mu:=\lambda \mu, \quad \lambda \dashv \nu:=\nu \lambda, \quad \lambda \dashv T_{j}^{i}:=\rho\left(\lambda^{(1)}\right)_{j}^{i} \lambda^{[\infty]}, \quad \lambda, \mu \in \mathcal{L}, \quad \nu \in \mathcal{L}_{o p} .
$$

define a right action of $\tilde{\mathcal{T}}$ on $\mathcal{L}$.

Proof. Formulas (18) define an action of the free algebra $k\left\langle T_{j}^{i}\right\rangle$ and actions of $\mathcal{L}$ and $\mathcal{L}_{o p}$. Thus, to prove the statement, it suffices to check that this action respects permutation rules of $\mathcal{L}$ and $\mathcal{L}_{o p}$ with the generators $T_{j}^{i}$ and annihilates the ideal $\mathcal{J}_{F R T}$. This is done by a straightforward calculation and left to the reader as an exercise. To give a hint, in the matrix form the action of $T$ can be written as $\lambda \dashv T=\delta(\lambda)$ (we suppressed $\rho$ ). Then the relations (17) will reduce to the equation $\tilde{S} \delta^{2}(\lambda)-\delta^{2}(\lambda) \tilde{S}=0$, which holds by the $\mathcal{H}$-invariance assumption (14).

The action (18) induces an $\mathcal{L}$-bimodule structure on $\mathcal{L}$ by $\alpha . \lambda . \beta=\lambda \dashv s(\beta) t(\alpha)$. By construction, this $\mathcal{L}$-bimodule structure coincides with the standard one. 
Lemma 3.3. The correspondence

$$
T_{j}^{i} \mapsto \sum_{k} T_{j}^{k} \otimes_{\mathcal{L}} T_{k}^{i} \quad \text { or } \quad T \mapsto T^{(1)} T^{(2)} \text { in the matrix form }
$$

defines a homomorphism of algebras $\Delta: \mathfrak{F}(T) \rightarrow \mathfrak{F}(T) \times_{\mathcal{L}} \mathfrak{F}(T)$, which is a homomorphism of $\mathcal{L}$-bimodules.

Proof. First of all notice that $\left(s(\lambda) T^{(1)}\right) T^{(2)}=T^{(1)}\left(t(\lambda) T^{(2)}\right)$, as follows from (16). Therefore $\Delta\left(T_{j}^{i}\right)$ belong to $\mathfrak{F}(T) \times_{\mathcal{L}} \mathfrak{F}(T)$. Extend $\Delta$ as a homomorphism to the free subalgebra $k\left\langle T_{j}^{i}\right\rangle \subset \mathfrak{F}(T)$. Extend $\Delta$ to $\mathcal{L} \subset \mathfrak{F}(T)$ and $\mathcal{L}_{o p} \subset \mathfrak{F}(T)$ as an $\mathcal{L}$-bimodule map:

$$
\Delta(\lambda)=1_{\mathfrak{F}(T)} \otimes_{\mathcal{L}} s(\lambda) \quad \text { and } \quad \Delta(\mu)=t(\mu) \otimes_{\mathcal{L}} 1_{\mathfrak{F}(T)}
$$

for $\lambda \in \mathcal{L}$ and $\mu \in \mathcal{L}_{o p}$. It is straightforward to check that the relations (5) are preserved by $\Delta$. Obviously $\Delta(\mathfrak{F}(T)) \subset \mathfrak{F}(T) \times_{\mathcal{L}} \mathfrak{F}(T)$, so the lemma is proven.

Proposition 3.4. (i) The coproduct $\Delta$ from Lemma 3.3 together with the anchor action (18) define a right $\mathcal{L}$-bialgebroid structure on the $\mathfrak{F}(T)$. (ii) The ideal $\mathcal{J}_{\text {dFRT }} \subset \mathfrak{F}(T)$ is a biideal, hence the projection from $\mathfrak{F}(T)$ along $\mathcal{J}_{\text {dFRT }}$ makes $\tilde{\mathcal{T}}$ a right $\mathcal{L}$-bialgebroid.

Proof. Statement (i) is obvious. Indeed, $\Delta$ is apparently coassociative. It is easy to see that the counit $\varepsilon$ defined through the anchor by

$$
\varepsilon(x):=1_{\mathcal{L}} \dashv x, \quad x \in \mathfrak{F}(T),
$$

satisfies (77).

Lemma 3.2 says that the ideal $\mathcal{J}_{d F R T}$ lies in the kernel of the anchor action (18). To prove (ii), we need to check consistency of the coproduct (19) with the multiplication in $\tilde{\mathcal{T}}$. An easy verification that relations (16) are respected is left to the reader. We will check only the dFRT relations (17). We have for $\Delta\left(\tilde{S}_{o p} T_{1} T_{2}\right)$, modulo $\mathcal{J}_{d F R T} \otimes_{\mathcal{L}} \mathfrak{F}(T)+\mathfrak{F}(T) \otimes_{\mathcal{L}} \mathcal{J}_{d F R T}$ :

$$
\begin{aligned}
\left(t\left(\tilde{S}_{o p}\right) T_{1}^{(1)} T_{2}^{(1)}\right)\left(T_{1}^{(2)} T_{2}^{(2)}\right) & =\left(T_{1}^{(1)} T_{2}^{(1)} s(\tilde{S})\right)\left(T_{1}^{(2)} T_{2}^{(2)}\right)=\left(T_{1}^{(1)} T_{2}^{(1)}\right)\left(t\left(\tilde{S}_{o p}\right) T_{1}^{(2)} T_{2}^{(2)}\right) \\
& =\left(T_{1}^{(1)} T_{2}^{(1)}\right)\left(T_{1}^{(2)} T_{2}^{(2)} s(\tilde{S})\right) .
\end{aligned}
$$

Here we set $t\left(\tilde{S}_{o p}\right):=(\mathrm{id} \otimes t)\left(S_{o p}\right)$ and $s(\tilde{S}):=(\mathrm{id} \otimes s)(S)$. We employed relations (17), then we pulled the coefficients of the matrix $s(\tilde{S})$ over to the right $\otimes_{\mathcal{L}}$-tensor factor to the rightmost position as the coefficients of the matrix $t(\tilde{S})$. After that we pushed the entries of $t(\tilde{S})$ to the left. This operation gave the matrix factor $t\left(\tilde{S}_{o p}\right)$. Applying relations (17) once again we obtained the final expression, which is equal to $\Delta\left(T_{1} T_{2} \tilde{S}\right)$.

Thus we proved that $\mathcal{J}_{d F R T}$ is a biideal in $\mathfrak{F}(T)$, hence the quotient $\tilde{\mathcal{T}}=\mathfrak{F}(T) / \mathcal{J}_{d F R T}$ inherits an $\mathcal{L}$-bialgebroid structure. 
Remark 3.5. In the constructions of the present subsection, we never used the dYBE on $\tilde{S}$, but only the $\mathcal{H}$-invariance (14). When $\mathcal{L}$ is the field of scalars, this construction gives the bialgebra associated with arbitrary matrix $\tilde{S}$, not necessarily as solution to the YBE, see FRT.

In what follows, we will need an extended dynamical FRT algebra whose description is given below. It has the well known analog in the theory of quantum groups, [FRT].

\subsection{Extended dFRT algebra}

In this subsection we construct a right bialgebroid $\underset{\mathcal{T}}{\tilde{S}} \underset{\mathcal{T}}{\tilde{\mathcal{T}}}$ generated by the double set of generators $\left\{T_{j}^{i}\right\},\left\{\bar{T}_{j}^{i}\right\}$ and its natural quotient bialgebroid. We restrict ourselves only with the description of $\tilde{\mathcal{T}} \underset{\tilde{S}}{\otimes} \tilde{\mathcal{T}}$ leaving verification of the bialgebroid axioms to the reader.

Instead of $k\left\langle T_{j}^{i}\right\rangle$, consider the free algebra $k\left\langle T_{j}^{i}, \bar{T}_{j}^{i}\right\rangle$ generated by the matrix elements of $\left\|T_{j}^{i}\right\|$ and $\left\|\bar{T}_{j}^{i}\right\|$ with the following $\mathcal{H} \otimes \mathcal{H}_{o p}$-module structure:

$$
x \triangleright T=T \rho(x), \quad y \triangleright T=\rho(y) T, \quad x \triangleright \bar{T}=\rho(\gamma(x)) \bar{T}, \quad y \triangleright \bar{T}=\bar{T} \rho(\gamma(y)),
$$

where $x \in \mathcal{H}$ and $y \in \mathcal{H}_{o p}$. The rest of the construction replicates the construction of the algebra $\tilde{\mathcal{T}}$ in the previous subsection. Using the $\mathcal{H}_{o p} \otimes \mathcal{H}$-coaction on $\mathcal{L}_{o p} \otimes \mathcal{L}$ and the $\mathcal{H}_{o p} \otimes \mathcal{H}$-module structure (21), we define the smash product $\mathfrak{F}(T, \bar{T})=k\left\langle T_{j}^{i}, \bar{T}_{j}^{i}\right\rangle \rtimes\left(\mathcal{L}_{o p} \otimes \mathcal{L}\right)$. There are the following commutation relations held in $\mathfrak{F}(T, \bar{T})$ :

$$
\begin{aligned}
\lambda T & =T \rho\left(\lambda^{(1)}\right) \lambda^{[\infty]}, & \lambda \bar{T} & =\rho\left(\gamma\left(\lambda^{(1)}\right)\right) \bar{T} \lambda^{[\infty]}, \\
\mu T & =\rho\left(\mu^{(1)}\right) T \mu^{[\infty]}, & \mu \bar{T} & =\bar{T} \rho\left(\gamma\left(\mu^{(1)}\right)\right) \mu^{[\infty]},
\end{aligned}
$$

where $\lambda \in \mathcal{L}$ and $\mu \in \mathcal{L}_{o p}$. There exist obvious algebra and anti-algebra embeddings, $s$ and $t$, from $\mathcal{L}$ to $\mathfrak{F}(T, \bar{T})$. The algebra $\mathfrak{F}(T, \bar{T})$ is endowed with an $\mathcal{L}$-bimodule structure via these maps and the right regular representation. We consider the two-sided ideal in $\mathfrak{F}(T, \bar{T})$ generated by the relations

$$
\tilde{S}_{o p} T_{1} T_{2}=T_{1} T_{2} \tilde{S}, \quad T_{2} \tilde{S} \bar{T}_{2}=\bar{T}_{1} \tilde{S}_{o p} T_{1}, \quad \tilde{S} \bar{T}_{2} \bar{T}_{1}=\bar{T}_{2} \bar{T}_{1} \tilde{S}_{o p}
$$

and denote by $\tilde{\mathcal{T}} \underset{\tilde{S}}{\otimes} \tilde{\mathcal{T}}$ the quotient of $\mathfrak{F}(T, \bar{T})$ by this ideal. We define the right anchor action of $\mathfrak{F}(T, \bar{T})$ on $\mathcal{L}$ setting

$$
\begin{array}{rlrl}
\lambda \dashv T_{j}^{i} & :=\rho\left(\lambda^{(1)}\right)_{j}^{i} \lambda^{[\infty]}, & \lambda \dashv \bar{T}_{j}^{i}:=\rho\left(\gamma\left(\lambda^{(1)}\right)\right)_{j}^{i} \lambda^{[\infty]}, \\
\lambda \dashv \mu & :=\lambda \mu, & \lambda \dashv \nu & :=\nu \lambda
\end{array}
$$


for $\lambda, \mu \in \mathcal{L}$ and $\nu \in \mathcal{L}_{o p}$. We can prove that this action descends to an action of the quotient

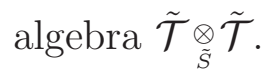

Then we introduce an $\mathcal{L}$-bimodule map from $\mathfrak{F}(T, \bar{T})$ to $\mathfrak{F}(T, \bar{T}) \times_{\mathcal{L}} \mathfrak{F}(T, \bar{T})$ setting it on the generators $\left\{T_{j}^{i}\right\}$ and $\left\{\bar{T}_{j}^{i}\right\}$ by

$$
\Delta\left(T_{j}^{i}\right)=\sum_{k} T_{j}^{k} \otimes_{\mathcal{L}} T_{k}^{i}, \quad \Delta\left(\bar{T}_{j}^{i}\right)=\sum_{k} \bar{T}_{k}^{i} \otimes_{\mathcal{L}} \bar{T}_{j}^{k} .
$$

or, in the matrix form, by

$$
\Delta(T)=T^{(1)} T^{(2)}, \quad \Delta(\bar{T})=\bar{T}^{(2)} \bar{T}^{(1)} .
$$

This correspondence is extended to the entire $\mathfrak{F}(T, \bar{T})$ as an algebra homomorphism. It is coassociative and defines, together with the anchor action (24), a right $\mathcal{L}$-bialgebroid structure on $\mathfrak{F}(T, \bar{T})$. The ideal of relations (23) is a biideal, hence the bialgebroid structure is carried over to $\tilde{\mathcal{T}} \underset{\tilde{S}}{\otimes} \tilde{\mathcal{T}}$.

The algebra $\underset{\mathcal{T}}{\otimes} \underset{\mathcal{S}}{\otimes} \tilde{\mathcal{T}}$ admits a natural quotient by the $\mathcal{H}$-invariant two-sided ideal specified by the relations

$$
\bar{T} T=1=T \bar{T}
$$

in the concise matrix form. We call this quotient extended dFRT algebra and denote by $\tilde{\mathcal{T}}_{\text {ext }}$. In fact, relations (27) define a biideal, so $\tilde{\mathcal{T}}_{\text {ext }}$ is a right $\mathcal{L}$-bialgebroid.

Remark 3.6. When one puts $\mathcal{H}=\mathcal{L}=k$, the dFRT and dRE algebras $\tilde{\mathcal{T}}$ and $\tilde{\mathcal{K}}$ degenerate to the ordinary FRT and RE algebras $\mathcal{T}$ and $\mathcal{K}$ associated with a matrix $S \in \operatorname{End}(V \otimes V)$.

\subsection{Category of comodules over a bialgebroid}

As was mentioned in Subsection 2.1 the category of modules over a bialgebroid is equipped with a monoidal structure. Let us render the definition of the monoidal category of comodules over a (right) bialgebroid following $\underline{\text { Sch }}$.

Definition 3.7. Let $\mathcal{B}$ be a right bialgebroid. An $\mathcal{L}$-bimodule $V$ is called right $\mathcal{B}$-comodule if it is equipped with an $\mathcal{L}$-bimodule map (coaction) $\delta: V \rightarrow V \otimes_{\mathcal{L}} \mathcal{B}$ fulfilling

1. for all $\lambda \in \mathcal{L}$ and $v \in V$

$$
\lambda . v^{[0]} \otimes_{\mathcal{L}} v^{(1)}=v^{[0]} \otimes_{\mathcal{L}} t_{\mathcal{B}}(\lambda) v^{(1)}, \quad \text { for } \quad v^{[0]} \otimes_{\mathcal{L}} v^{(1)}=\delta(v),
$$


2. coassociativity condition

$$
\left(\delta \otimes_{\mathcal{L}} \mathrm{id}\right) \circ \delta=\left(\mathrm{id} \otimes_{\mathcal{L}} \Delta\right) \circ \delta
$$

3. counital condition: (id $\left.\otimes_{\mathcal{L}} \varepsilon_{\mathcal{B}}\right) \circ \delta \simeq$ id under the identification $V \otimes_{\mathcal{L}} \mathcal{L} \simeq V$.

All $\mathcal{B}$-comodules form a category, $\mathcal{C}$. The set $\operatorname{Hom}_{\mathcal{C}}(V, W)$ consists of maps $V \stackrel{\phi}{\rightarrow} W$ such that the diagram

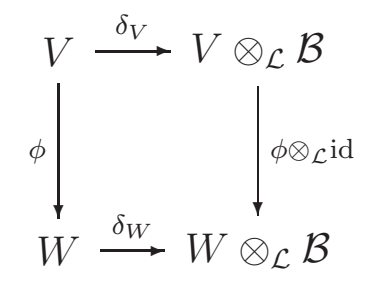

is commutative.

Let us introduce on $\mathcal{C}$ a structure of strict monoidal category. Given two $\mathcal{B}$-comodules $V, W$ define the $\mathcal{B}$-coaction on $V \otimes_{\mathcal{L}} W$ as

$$
v \otimes_{\mathcal{L}} w \mapsto v^{[0]} \otimes_{\mathcal{L}} w^{[0]} \otimes_{\mathcal{L}} v^{(1)} w^{(1)}
$$

This map is correctly defined by virtue of (28) and fulfills the axioms of coaction. The tensor product of two morphisms, $\phi$ and $\psi$, is set to be $\phi \otimes_{\mathcal{L}} \psi$. The unit object in $\mathcal{C}$ is $\mathcal{L}$, with the coaction $\lambda \mapsto \lambda \otimes_{\mathcal{L}} 1_{\mathcal{B}}$. By construction, the category $\mathcal{C}$ has a forgetful functor to the category of $\mathcal{L}$-bimodules, and this functor is strong monoidal.

Let $\mathcal{A}$ be an $\mathcal{L}$-bimodule algebra. Then

$$
\mathcal{A} \times_{\mathcal{L}} \mathcal{B}=\left\{z \in \mathcal{A} \otimes_{\mathcal{L}} \mathcal{B} \mid(\lambda . \otimes 1) z=\left(1 \otimes t_{\mathcal{B}}(\lambda)\right) z, \forall \lambda \in \mathcal{L}\right\}
$$

is again an $\mathcal{L}$-bimodule algebra. If $\mathcal{A}$ is a $\mathcal{B}$-comodule, then the coaction takes values in $\mathcal{A} \times{ }_{\mathcal{L}} \mathcal{B}$. An $\mathcal{L}$-bimodule algebra and $\mathcal{B}$-comodule is an algebra in the category $\mathcal{C}$ if and only if the coaction $\delta: \mathcal{A} \rightarrow \mathcal{A} \times_{\mathcal{L}} \mathcal{B}$ is a homomorphism of $\mathcal{L}$-bimodule algebras.

Definition 3.8. An algebra in $\mathcal{C}$ is called $\mathcal{B}$-comodule algebra.

Clearly the bialgebroid $\mathcal{B}$ itself is a $\mathcal{B}$-comodule algebra.

\section{4 dRE algebra associated with a dynamical matrix $\tilde{S}$}

This time denote the dual base to $\left\{e_{i}^{j} \subset \operatorname{End}(V)\right\}$ by $\left\{K_{j}^{i}\right\} \subset \operatorname{End}^{*}(V)$. The left $\mathcal{H}$-action

$$
h \triangleright K=\rho\left(\gamma\left(h^{(1)}\right)\right) K \rho\left(h^{(2)}\right), \quad \text { where } \quad K:=\sum_{i, j} e_{i}^{j} \otimes K_{j}^{i},
$$


on $\operatorname{End}^{*}(V)$ naturally extends to a left action on $k\left\langle K_{j}^{i}\right\rangle$ making it an $\mathcal{H}$-module algebra. Consider the twisted tensor product of Hopf algebras $\mathcal{H} \otimes \mathfrak{D H}$ and construct the corresponding twisted module algebra $\mathfrak{F}(K):=k\left\langle K_{j}^{i}\right\rangle \rtimes \mathcal{L}$. The algebra $\mathfrak{F}(K)$ contains $\mathcal{L}$ as a subalgebra and thus it is a natural $\mathcal{L}$-bimodule. The elements of $\mathcal{L} \subset \mathfrak{F}(K)$ obey the following permutation rules with the generators $K_{j}^{i}$ :

$$
\lambda K=\rho\left(\gamma\left(\lambda^{(1)}\right)\right) K \rho\left(\lambda^{(2)}\right) \lambda^{[\infty]} .
$$

The actions of $\mathcal{H}$ and $\mathcal{L}$ on $\mathfrak{F}(K)$ give rise to an action of the left $\mathcal{L}$-bialgebroid $\mathcal{L} \rtimes \mathcal{H}$. In fact, $\mathfrak{F}(K)$ is an algebra over $\mathcal{L} \rtimes \mathcal{H}$.

Definition 3.9. The dRE algebra associated with an $\mathcal{H}$-invariant element $\tilde{S} \in \operatorname{End}^{\otimes 2}(V) \otimes \mathcal{L}$ and denoted further by $\tilde{\mathcal{K}}$ is the quotient of $\mathfrak{F}(K)$ by the relations

$$
\tilde{S} K_{2} \tilde{S} K_{2}=K_{2} \tilde{S} K_{2} \tilde{S}
$$

Note with care that the coefficients of $\tilde{S}$ belong to $\mathcal{L}$ and do not commute with $K_{j}^{i}$.

One can check, using (14), that the ideal in $\mathfrak{F}(K)$ generated by (32) is invariant with respect to $\mathcal{H}$, so $\tilde{\mathcal{K}}$ is an $\mathcal{H}$-module algebra. It is also is an $\mathcal{L}$-bimodule algebra whose $\mathcal{L}$ bimodule structure is induced by the homomorphism $\mathcal{L} \rightarrow \tilde{\mathcal{K}}$ denoted further $s_{\tilde{\mathcal{K}}}$. These two structures make $\tilde{K}$ a module algebra over the bialgebroid $\mathcal{L} \rtimes \mathcal{H}$.

Proposition 3.10. The correspondence $\delta_{\tilde{\mathcal{K}}}: K_{j}^{i} \otimes \lambda \mapsto \sum_{\alpha, \beta}\left(K_{\beta}^{\alpha} \otimes 1_{\mathcal{L}}\right) \otimes_{\mathcal{L}}\left(\bar{T}_{j}^{\beta} T_{\alpha}^{i} \otimes 1_{\mathcal{L}_{o p}} \otimes \lambda\right)$ defines on $\tilde{\mathcal{K}}$ a structure of right $\tilde{\mathcal{T}} \underset{\tilde{S}}{\otimes} \tilde{\mathcal{T}}$-comodule algebra. It also makes $\tilde{\mathcal{K}}$ a right $\tilde{\mathcal{T}}_{\text {ext }}{ }^{-}$ comodule algebra via the projection $\tilde{\mathcal{T}}_{\tilde{S}}^{\otimes} \tilde{\mathcal{T}} \rightarrow \tilde{\mathcal{T}}_{\text {ext }}$.

Proof. Put $\mathcal{A}:=\tilde{\mathcal{K}}$ and $\mathcal{B}:=\tilde{\mathcal{T}}{ }_{\tilde{S}} \tilde{\mathcal{T}}$. Let us show that the image of the "similarity transformation" $\delta_{\tilde{\mathcal{K}}}: K \mapsto \bar{T} K T$ lies in $\operatorname{End}(V) \otimes \mathcal{A} \times{ }_{\mathcal{L}} \mathcal{B}$. Indeed,

$$
\begin{aligned}
\bar{T} s_{\mathcal{A}}(\lambda) K T & =\bar{T} \rho\left(\gamma\left(\lambda^{(1)}\right)\right) K \rho\left(\lambda^{(2)}\right) s_{\mathcal{A}}\left(\lambda^{[\infty]}\right) T \\
& =\bar{T} \rho\left(\left(\gamma\left(\lambda^{(1)}\right)\right) K \rho\left(\lambda^{(2)}\right) T t_{\mathcal{B}}\left(\lambda^{[\infty]}\right)=t_{\mathcal{B}}(\lambda) \bar{T} K T .\right.
\end{aligned}
$$

The first and the last equalities are obtained using (31) and (22). In the middle one, we employed the definition of tensor product over $\mathcal{L}$.

Next we prove that $\delta_{\tilde{\mathcal{K}}}$ defines an algebra homomorphism from $\mathcal{A}$ to $\mathcal{A} \times{ }_{\mathcal{L}} \mathcal{B}$. First of all, observe that $s_{\mathcal{B}}(\lambda)(\bar{T} K T)=\rho\left(\gamma \lambda^{(1)}\right)(\bar{T} K T) \rho\left(\lambda^{(2)}\right) s_{\mathcal{B}}\left(\lambda^{[\infty]}\right)$. Thus the relation (B1) is preserved. 
Let us compute the expression $s_{\mathcal{B}}(\tilde{S})\left(\bar{T}_{2} K_{2} T_{2}\right) s_{\mathcal{B}}(\tilde{S})\left(\bar{T}_{2} K_{2} T_{2}\right)$. Using (17) twice, we find it equal to

$$
\begin{aligned}
s_{\mathcal{B}}(\tilde{S})\left(\bar{T}_{2} K_{2} T_{2}\right) s_{\mathcal{B}}(\tilde{S})\left(\bar{T}_{2} K_{2} T_{2}\right) & =s_{\mathcal{B}}(\tilde{S}) \bar{T}_{2} K_{2} \bar{T}_{1} t_{\mathcal{B}}\left(\tilde{S}_{o p}\right) T_{1} K_{2} T_{2}= \\
=s_{\mathcal{B}}(\tilde{S}) \bar{T}_{2} \bar{T}_{1} K_{2} t_{\mathcal{B}}\left(\tilde{S}_{o p}\right) K_{2} T_{1} T_{2} & =\bar{T}_{2} \bar{T}_{1} t_{\mathcal{B}}\left(\tilde{S}_{o p}\right) K_{2} t_{\mathcal{B}}\left(\tilde{S}_{o p}\right) K_{2} T_{1} T_{2}
\end{aligned}
$$

We can replace $t_{\mathcal{B}}\left(\tilde{S}_{o p}\right)$ by $s_{\mathcal{A}}(\tilde{S})$ in the last expression. Indeed, pull the entries of the right matrix $t_{\mathcal{B}}\left(\tilde{S}_{o p}\right)$ to the right-most position. We have to commute them with the entries of the matrices $T_{1}$ and $T_{2}$, according to (22) . Then we replace the entries of $t_{\mathcal{B}}(S)$ by the entries of $s_{\mathcal{A}}(S)$, using tensoring over $\mathcal{L}$, and pull them back to the left through the entries of $K_{2}$. This produces the desired effect for right $t_{\mathcal{B}}\left(\tilde{S}_{o p}\right)$. We do the same with the left matrix $t_{\mathcal{B}}\left(\tilde{S}_{o p}\right)$. This time it is a bit more complicated, because we have to pass through $s_{\mathcal{A}}(S)$ obtained in the previous step and through another matrix $K_{2}$. The result will be the same: the left matrix $t_{\mathcal{B}}\left(\tilde{S}_{o p}\right)$ is replaced by $s_{\mathcal{A}}(\tilde{S})$. Thus (133) transforms into

$$
\bar{T}_{2} \bar{T}_{1} s_{\mathcal{A}}(\tilde{S}) K_{2} s_{\mathcal{A}}(\tilde{S}) K_{2} T_{1} T_{2}=\bar{T}_{2} \bar{T}_{1} K_{2} s_{\mathcal{A}}(\tilde{S}) K_{2} s_{\mathcal{A}}(\tilde{S}) T_{1} T_{2}
$$

where we used (32). Next we act in the reversed direction and replace $s_{\mathcal{A}}(\tilde{S})$ by $t_{\mathcal{B}}\left(\tilde{S}_{\text {op }}\right)$. Then (33) becomes

$$
\bar{T}_{2} \bar{T}_{1} K_{2} t_{\mathcal{B}}\left(\tilde{S}_{o p}\right) K_{2} t_{\mathcal{B}}\left(\tilde{S}_{o p}\right) T_{1} T_{2}=\bar{T}_{2} K_{2} \bar{T}_{1} t_{\mathcal{B}}\left(\tilde{S}_{o p}\right) T_{1} K_{2} T_{2} s_{\mathcal{B}}(\tilde{S})
$$

This is equal to $\left(\bar{T}_{2} K_{2} T_{2}\right) s_{\mathcal{B}}(\tilde{S})\left(\bar{T}_{2} K_{2} T_{2}\right) s_{\mathcal{B}}(\tilde{S})$, so the relations (32) are preserved.

We have shown that $\delta_{\tilde{\mathcal{K}}}$ is an algebra homomorphism. To finish the proof, we must check conditions 2 and 3 of Definition 3.7 . This is an easy and straightforward exercise.

\section{Twisting the FRT and RE algebras}

The remainder of the paper is devoted to the study of the dFRT and dRE algebras when the matrix $\tilde{S}$ is obtained from a universal dynamical R-matrix. That R-matrix itself is assumed to be a dynamical twist of a quasitriangular structure on a Hopf algebra, $\mathcal{U}$. Our gual is to show that the dFRT and dRE algebras are related to the ordinary FRT and RE algebras by certain dynamical twists. As to the dFRT algebra, this is more or less straightforward and can be readily extracted from [DM2]. The case of dRE algebra is not obvious, so this will be in the focus of our attention. For better understanding of what is going on in the dynamical situation we first take a look at how a Hopf algebra twist transforms the ordinary FRT and RE algebras. 


\subsection{Twist and twisted tensor square}

Let $\mathcal{U}$ be a quasitriangular Hopf algebra with the universal R-matrix $\mathcal{R}$. Let $\mathcal{U}^{o p}$ denote the coopposite Hopf algebra. The FRT algebra is a module over $\mathcal{U}^{o p} \otimes \mathcal{U}$. Recall from [DM3] that the RE algebra a module over $\mathcal{U}^{\mathcal{R}} \mathcal{U}$, the twisted tensor square of $\mathcal{U}$, [RS. Those two Hopf algebras are related by the composition of twists:

$$
\mathcal{U}^{o p} \otimes \mathcal{U} \stackrel{\mathcal{R}_{13}}{\longrightarrow} \mathcal{U} \otimes \mathcal{U} \stackrel{\mathcal{R}_{23}}{\longrightarrow} \mathcal{U}^{\mathcal{R}} \mathcal{U}
$$

where $\mathcal{R}_{i j} \in(\mathcal{U} \otimes \mathcal{U}) \otimes(\mathcal{U} \otimes \mathcal{U})$. This composite twist transforms the FRT algebra to the RE one. So the latter is a twist of a module algebra over the intermediate Hopf algebra $\mathcal{U} \otimes \mathcal{U}$.

Now suppose $\mathcal{F}$ is a twisting cocycle in $\mathcal{U}$, i.e. an invertible element from $\mathcal{U} \otimes \mathcal{U}$ satisfying

$$
\mathcal{F}_{(12) 3} \mathcal{F}_{12}=\mathcal{F}_{1(23)} \mathcal{F}_{23}, \quad \varepsilon_{1}(\mathcal{F})=\varepsilon_{2}(\mathcal{F})=1_{\mathcal{U} \otimes 2}
$$

Two twists $\mathcal{F}^{\{i\}}, i=1,2$, are called gauge equivalent if there is an invertible element $v \in \mathcal{U}$ such that

$$
\Delta(v) \mathcal{F}^{\{1\}}=\mathcal{F}^{\{2\}}(v \otimes v)
$$

If $\tilde{\mathcal{U}}^{\{i\}}, i=1,2$, be respectively the twists of $\mathcal{U}$ by $\tilde{\mathcal{F}}^{\{i\}}$, then the conjugation $x \mapsto v x \bar{v}$ implements a Hopf algebra isomorphism $\tilde{\mathcal{U}}^{\{1\}} \rightarrow \tilde{\mathcal{U}}^{\{2\}}$.

Given a twisting cocycle $\mathcal{F}$ of $\mathcal{U}$, we can consider the following two twists $\Psi^{\{i\}}, i=1,2$, of the Hopf algebra $\mathcal{U} \otimes \mathcal{U}$. First we take the twist by $\mathcal{R}_{23} \in(\mathcal{U} \otimes \mathcal{U}) \otimes(\mathcal{U} \otimes \mathcal{U})$; then the coproduct $\Delta: \mathcal{U} \rightarrow \mathcal{U}^{\mathcal{R}} \mathcal{U}$ is a Hopf algebra map and sends the $\mathcal{U}$-cocycle $\mathcal{F}$ to the $\mathcal{U}^{\circledR} \mathcal{U}$ cocycle $\mathcal{F}_{(12)(34)}$. We put $\Psi^{\{1\}}:=\mathcal{F}_{13} \mathcal{F}_{24} \tilde{\mathcal{R}}_{23}$. The other twist is the composition of the twists $\mathcal{F} \otimes \mathcal{F}$ and $\tilde{\mathcal{R}}_{23}=\mathcal{F}_{32} \mathcal{R}_{23} \mathcal{F}_{23}$, thus we put $\Psi^{\{2\}}:=\mathcal{R}_{23} \mathcal{F}_{(12)(34)}$.

The question is how $\Psi^{\{1\}}$ and $\Psi^{\{2\}}$ are related to each other. The answer is simple but very important for further considerations.

Proposition 4.1. The twists $\Psi^{\{1\}}$ and $\Psi^{\{2\}}$ are gauge equivalent:

$$
\mathcal{F}_{(13)(24)} \Psi^{\{1\}}=\Psi^{\{2\}} \mathcal{F}_{12} \mathcal{F}_{34}
$$

Proof. Formula (36) is a specialization of the formula (35) for $\mathcal{U} \otimes \mathcal{U}$ instead of $\mathcal{U}$, with $v=\mathcal{F}$. By definition of $\Psi^{\{1\}}$ and $\Psi^{\{2\}}$, equation (36) is nothing else than

$$
\mathcal{F}_{(13)(24)} \mathcal{F}_{13} \mathcal{F}_{24} \tilde{\mathcal{R}}_{23}=\mathcal{R}_{23} \mathcal{F}_{(12)(34)} \mathcal{F}_{12} \mathcal{F}_{34}
$$

But this latter equation is a corollary of the identity $\mathcal{F}_{(12)(34)} \mathcal{F}_{12} \mathcal{F}_{34}=\mathcal{F}_{1(234)} \mathcal{F}_{2(34)} \mathcal{F}_{34}=$ $\mathcal{F}_{(123) 4} \mathcal{F}_{(12) 3} \mathcal{F}_{12}$ following from the twisting cocycle equation (34). 


\subsection{How a twist of the Hopf algebra affects the RE algebra}

We are going to obtain the dRE algebra by a dynamical twist of the ordinary RE algebra. To develop a strategy of solving this problem, we take a closer look at how the RE algebra behaves under a non-dynamical or ordinary Hopf algebra twist. The following diagram displays the relations between the FRT and RE algebras associated with a quasitriangular Hopf algebra $(\mathcal{U}, \mathcal{R})$ and their transformations under the twist $\mathcal{F}$ of $\mathcal{U}$.

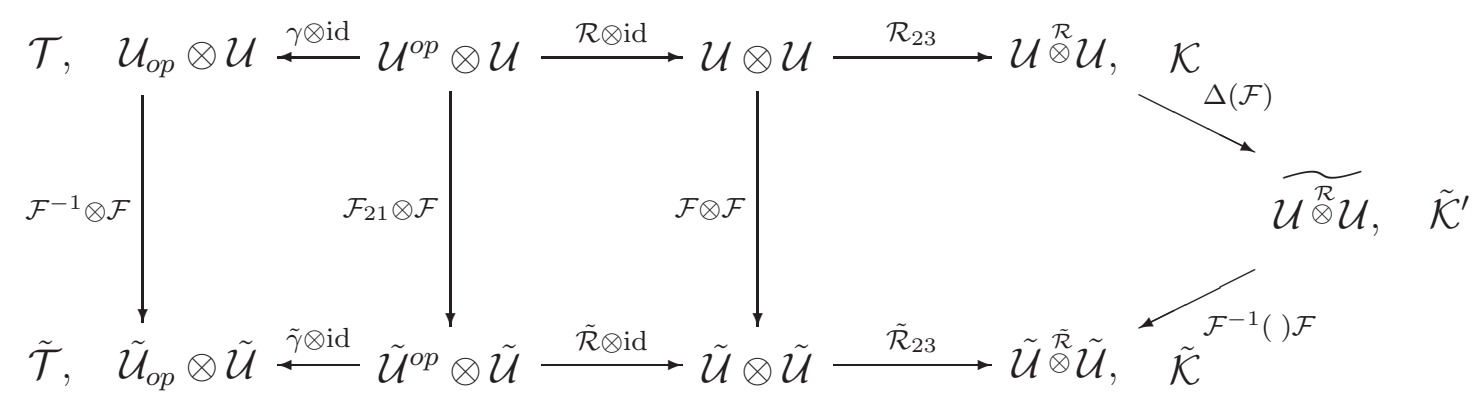

The rightmost vertex of this diagram exists due to Proposition 4.1] Its outgoing arrow denotes the coboundary twist with $\mathcal{F}$ considered as an element of $\mathcal{U}^{\circledR} \mathcal{R}$

In case of dynamical twist $\mathcal{F}$, we cannot draw exactly the same diagram. We can construct dFRT algebra as a "bimodule" over certain quantum groupoid extending $\mathcal{U}$ (see Subsection (7.2), but there are two severe obstructions to further proceeding to the dRE algebra. First, the notion of bialgebroid antipode is not obvious. Second, and this is crucial, there is no natural construction of the twisted tensor square for quasitriangular bialgebroids. Thus we cannot reach the right bottom corner moving counterclockwise from the left upper one.

Within the Hopf algebra setting, there is a way of getting $\tilde{\mathcal{K}}$ directly from $\mathcal{K}$ by applying two consecutive twists. Namely, starting from the right upper corner and moving through the right-most vertex. In this passage, essential is the first twist, because the second one is coboundary and results in an isomorphism of the module algebras. The first or essential twist may well be constructed out of a dynamical cocycle $\mathcal{F}$ thus yielding an algebra, $\tilde{\mathcal{K}}^{\prime}$, that may be called a dynamical RE algebra. Our goal is to prove that this algebra is a specific case of the dRE algebra introduced in Subsection 3.4. To solve this problem, we should find a set of generators in $\tilde{\mathcal{K}}^{\prime}$ satisfying relations (31) and (32) with $\tilde{S}$ being the image of the universal dynamical R-matrix multiplied by the matrix permutation.

Let us consider in more detail how the RE transforms under the ordinary twist. The RE algebra is commutative with respect to the universal R-matrix of $\mathcal{U}^{\mathcal{R}} \mathcal{U}$, and the RE relations are the corollary of this fact. The universal R-matrix of $\mathcal{U}^{\mathcal{R}} \mathcal{U}$ is expressed through $\mathcal{R}$ and $\mathcal{R}^{-}:=\mathcal{R}_{21}^{-1}$ by $\mathcal{R}_{12}^{-} \mathcal{R}_{24} \mathcal{R}_{13}^{-} \mathcal{R}_{23}$. The transition from $\mathcal{U}^{\mathcal{R}} \mathcal{U}$ to $\widetilde{\mathcal{U}^{\mathcal{R}} \mathcal{U}}$ destroys the RE relations 
in $\tilde{\mathcal{K}}^{\prime}$. One reason for that is the R-matrix of $\widetilde{\mathcal{U}^{\mathcal{R}} \mathcal{U}}$ loosing its factorized form under this twist. This problem can be fixed by the subsequent coboundary twist from Proposition 4.1. This transformation restores the desired factorized form $\tilde{\mathcal{R}}_{12}^{-} \tilde{\mathcal{R}}_{24} \tilde{\mathcal{R}}_{13}^{-} \tilde{\mathcal{R}}_{23}$ of the universal R-matrix. However this is not sufficient. While $\mathcal{U}^{\mathcal{R}} \mathcal{U}$ and $\tilde{\mathcal{U}} \otimes \tilde{\mathcal{U}}$ coincide with $\mathcal{U} \otimes \mathcal{U}$ as associative algebras, $\mathcal{K}$ and $\tilde{\mathcal{K}}$ are different (although isomorphic) as $\mathcal{U} \otimes \mathcal{U}$-modules. Indeed, the action involves antipode, which is changed under the twist by a similarity transformation. Recall that the new antipode have the form $\tilde{\gamma}(h)=\gamma(\bar{\zeta} h \zeta)$, where $\zeta=\overline{\mathcal{F}}_{2} \bar{\gamma}\left(\overline{\mathcal{F}}_{1}\right)$, Dr3]. Therefore, if we perform the coboundary twist of $\tilde{\mathcal{U}} \tilde{\mathcal{R}}^{\tilde{\mathcal{U}}}$ induced by $\zeta$, we bring the $\mathcal{U} \otimes \mathcal{U}$-module structure of $\tilde{\mathcal{K}}$ right to that of $\mathcal{K}$.

Summarizing, in the Hopf algebra case, the algebra $\tilde{\mathcal{K}}$ can be obtained from $\mathcal{K}$ by the twist $(\Delta)(\mathcal{F})$ of the Hopf algebra $\mathcal{U}^{\mathcal{R}} \mathcal{U}$ and the subsequent coboundary twist induced by

$\mathcal{F}(\zeta \otimes \mathrm{id}) \in \mathcal{U}^{\mathcal{R}} \mathcal{U}$. The first twist naturally carries over to the dynamical situation. It turns out that the coboundary twist also has a dynamical version. It will be constructed in Sections 6 and 7.

\section{$5 \quad$ Algebra of symmetric dynamical tensors}

The present section is of technical character. Here we study subspaces in $\mathcal{U}^{\otimes n} \otimes \mathcal{L}$ possessing certain symmetries with respect to the Hopf algebra $\mathcal{H} \subset \mathcal{U}$.

\subsection{Some definitions and notation}

In the sequel of the paper we fix a quasitriangular Hopf algebra $\mathcal{U} \supset \mathcal{H}$ with the universal R-matrix $\mathcal{R}$. We will pursue a thorough study of a dynamical twist $\mathcal{F}$ over an $\mathcal{H}$-base algebra $\mathcal{L}$ with values in $\mathcal{U}$ and its certain derived twists. When applied to the ordinary FRT and RE algebras related to $\mathcal{U}$, those twists will be shown to produce the dFRT an dRE algebras introduced in Section 3, To this end, we need to develop a certain algebraic machinery.

Let us introduce notation $\check{\mathcal{U}}:=\mathcal{U} \otimes \mathcal{L}$ and $\check{\mathcal{U}}^{n}:=\mathcal{U}^{\otimes n} \otimes \mathcal{L}$ for $n=2,3 \ldots$. It is convenient to put $\check{\mathcal{U}}^{0}:=\mathcal{L}$. Elements of $\check{\mathcal{U}}^{n}$ will be called dynamical tensors of rank $n$. We will use the following convention: $\mathcal{U}$-factors of $\check{\mathcal{U}}^{n}$ are labelled by positive integers; the $\mathcal{L}$-factor is not indicated explicitly, assumed to be always on the right. $\mathcal{U}$-factors stemming from the $\mathcal{H}$-coaction $\delta$ with the subsequent embedding to $\mathcal{U}$ are marked by the right group of subscripts separated by |, for example

$u_{1 \mid 2}:=u_{1} \otimes u_{\mathcal{L}}^{(1)} \otimes u_{\mathcal{L}}^{[\infty]}, \quad u_{(12) \mid 3}:=u_{1}^{(1)} \otimes u_{1}^{(2)} \otimes u_{\mathcal{L}}^{(1)} \otimes u_{\mathcal{L}}^{[\infty]}, \quad u_{31 \mid 42}:=u_{2} \otimes u_{\mathcal{L}}^{(2)} \otimes u_{1} \otimes u_{\mathcal{L}}^{(1)} \otimes u_{\mathcal{L}}^{[\infty]}$. 
Let $\mathfrak{S}_{n}$ be the symmetric group of permutations of $n$-tuples. It naturally acts on $\check{\mathcal{U}}^{n}$ by permutations of tensor factors. Denote by $\epsilon$ the unit element of $\mathfrak{S}_{n}$ and by $\tau$ the flip $(1 \ldots n) \mapsto(n \ldots 1)$. For any pair $\alpha, \beta \in \mathfrak{S}_{n}$, select in $\check{\mathcal{U}}^{n}$ the following subspace of $\mathcal{H}$ invariant elements:

$$
{ }_{\alpha} \check{\mathcal{U}}_{\beta}{ }^{n}:=\left\{u_{1 \ldots n} \otimes u_{\mathcal{L}} \in \check{\mathcal{U}}^{n} \mid\left(\Delta_{\alpha}^{n}\right)\left(h^{(1)}\right) u_{1 \ldots n} \otimes h^{(2)} \triangleright u_{\mathcal{L}}=u_{1 \ldots n}\left(\Delta_{\beta}^{n}\right)(h) \otimes u_{\mathcal{L}}, \forall h \in \mathcal{H}\right\} .
$$

Here $\Delta_{\alpha}^{n}:=\alpha \circ \Delta^{n}, \alpha \in \mathfrak{S}_{n}$, and $\Delta^{n}$ is the $n$-fold coproduct.

We will say that a dynamical tensor of rank $n$ has definite type of symmetry or just symmetric dynamical tensor if it belongs to ${ }_{\alpha} \check{\mathcal{U}}_{\beta}{ }^{n}$ for some $(\alpha, \beta) \in \mathfrak{S}_{n} \times \mathfrak{S}_{n}$. There is a partial associative operation on the subspace of symmetric dynamical tensors, which restricts from the multiplication in $\check{\mathcal{U}}^{n}$. We have, ${ }_{\alpha} \check{\mathcal{U}}_{\beta}{ }_{\beta} \check{\mathcal{U}}_{\sigma}{ }^{n} \subset{ }_{\alpha} \check{\mathcal{U}}_{\sigma}{ }^{n}$ for all $\alpha, \beta, \sigma \in \mathfrak{S}_{n}$. The $\mathfrak{S}_{n} \times \mathfrak{S}_{n}$-graded linear space $\check{\mathcal{U}}_{s y m}^{n}=\oplus_{\alpha, \beta \in \mathfrak{S}_{n} \alpha} \check{\mathcal{U}}_{\beta}{ }^{n}$ together with this partial multiplication is called algebra of symmetric dynamical tensors of rank $n$. Clearly ${ }_{\alpha} \check{\mathcal{U}}_{\alpha}{ }^{n}$ for any $\alpha \in \mathfrak{S}_{n}$ is a unital associative algebra in the usual sense. We consider the space $\check{\mathcal{U}}^{n}$ of all dynamical tensors as a "two-sided module" over $\check{\mathcal{U}}_{\text {sym }}^{n}$.

It is possible to introduce a structure of associative algebra on the infinite direct sum $\oplus_{m=0}^{\infty} \check{\mathcal{U}}_{s y m}^{m}$ which is a kind of tensor product. We will mostly use only its restriction to $\oplus_{m=0}^{\infty} \check{\mathcal{U}}_{\epsilon}^{m}$.

Proposition 5.1. Let $i, k, n$ be positive integers such that $i+k \leq n$. Then the embedding

$$
\iota_{i}: \check{\mathcal{U}}^{k} \rightarrow \check{\mathcal{U}}^{n}, \quad \iota_{i}: u_{1 \ldots k} \mapsto u_{i \ldots i+k-1 \mid i+k \ldots n}
$$

induces an embedding $\check{\mathcal{U}}_{\epsilon}^{k} \rightarrow \check{\epsilon}_{\epsilon}{ }^{n}$. The map

$$
\check{\mathcal{U}}^{i} \otimes \check{\mathcal{U}}^{k} \rightarrow \check{\mathcal{U}}^{i+k}, \quad u \otimes v \mapsto \iota_{1}(u) \iota_{i+1}(v)=\iota_{i+1}(v) \iota_{1}(u), \quad i, k=0,1 \ldots
$$

defines an associative operation (tensor product) on $\oplus_{m=0}^{\infty} \check{\mathcal{U}}_{\epsilon}{ }^{m}$.

Proof. The embedding (37) is obvious. Due to (19), elements from ${ }_{\epsilon} \check{\mathcal{U}}_{\epsilon}^{k}$ commute with $\delta^{k}(\mathcal{L})$. Hence the image $\iota_{1}\left(\check{\mathcal{U}}^{i}\right)$ commutes with $\iota_{i+1}\left(\check{\mathcal{U}}_{\epsilon}{ }^{k}\right)$ in (38). Associativity of the operation (38) follows from associativity and coassociativity of $\delta$.

Remark 5.2. Note that symmetric dynamical tensors of type $(\epsilon, \epsilon)$ yield morphisms in dynamical categories of [DM1]. The tensor product from Proposition 5.1] is induced by the tensor product of morphisms. 


\subsection{Dynamical twist and R-matrix}

Let $\mathcal{U}$ be a Hopf algebra containing $\mathcal{H}$ as a Hopf subalgebra. A dynamical $(\mathcal{U}, \mathcal{H}, \mathcal{L})$-twist is an element $\mathcal{F} \in \breve{\mathcal{U}}_{\epsilon}{ }^{2}$ satisfying the conditions

$$
\begin{gathered}
\mathcal{F}_{(12) 3} \mathcal{F}_{12 \mid 3}=\mathcal{F}_{1(23)} \mathcal{F}_{23}, \\
\varepsilon_{1}(\mathcal{F})=1_{\check{\mathcal{U}}^{2}}=\varepsilon_{2}(\mathcal{F}) .
\end{gathered}
$$

Numerous examples of dynamical twists over various bases relative both to classical and quantum groups are built in [EV1, ESS, EE, EEM].

The notion of coboundary twist can be modified for the dynamical situation. Specifically, two twists $\mathcal{F}^{\{i\}} \in{ }_{\epsilon} \check{\mathcal{U}}_{\epsilon}{ }^{2}, i=1,2$, are called gauge equivalent if there exists $\zeta \in{ }_{\epsilon} \check{\mathcal{U}}_{\epsilon}$ such that

$$
\Delta(\zeta) \mathcal{F}^{\{1\}}=\mathcal{F}^{\{2\}} \zeta_{1 \mid 2} \zeta_{2}
$$

Note that each factor in this equality has the symmetry type $(\epsilon, \epsilon)$; so this is an equality in the associative algebra $\check{\epsilon}_{\epsilon}^{2}$. Also note that two $\zeta$-factors on the right commute with each other, see (38).

Observe that the R-matrix $\mathcal{R}$ of the Hopf algebra $\mathcal{U}$ belongs to ${ }_{\tau} \check{\mathcal{U}}_{\epsilon}^{2}$. The element $\overline{\mathcal{F}}_{21} \mathcal{R F} \in{ }_{\tau} \check{\mathcal{U}}_{\epsilon}^{2}$ is called dynamical R-matrix and satisfies the (dynamical Yang-Baxter) equation

$$
\tilde{\mathcal{R}}_{12} \tilde{\mathcal{R}}_{13 \mid 2} \tilde{\mathcal{R}}_{23}=\tilde{\mathcal{R}}_{23 \mid 1} \tilde{\mathcal{R}}_{13} \tilde{\mathcal{R}}_{12 \mid 3} .
$$

Note that each factor in (41) has a definite symmetry type in $\check{\mathcal{U}}_{\text {sym }}^{3}$, thus (41) is an equation in $\check{\mathcal{U}}_{\text {sym }}^{3}$.

The gauge transformation of two dynamical twist corresponding to the element $\zeta \in{ }_{\epsilon} \check{\mathcal{U}}_{\epsilon}$ amounts to the transformation of a dynamical R-matrix

$$
\tilde{\mathcal{R}} \mapsto \bar{\zeta}_{2 \mid 1} \bar{\zeta}_{1} \tilde{\mathcal{R}} \zeta_{1 \mid 2} \zeta_{2}
$$

The dynamical R-matrix $\tilde{\mathcal{R}}$ defines braiding in certain monoidal categories, DM1, DM2].

\subsection{One technical lemma}

Denote $\omega=\Theta_{1}^{-1} \gamma^{-1}\left(\Theta_{2}^{-1}\right)$, a Drinfeld element of the double Hopf algebra $\mathfrak{D H}$, Dr2]. It satisfies the equality $\Delta\left(\omega^{-1}\right)(\omega \otimes \omega)=\Theta^{-1} \Theta_{21}^{-1}$. From this we conclude that the map $\lambda \mapsto \omega \triangleright \lambda$ is an automorphism of the base algebra $\mathcal{L}$ (recall that in terms of $\Theta$ the condition 
(9) reads $\left(\Theta_{2} \triangleright \lambda\right)\left(\Theta_{1} \triangleright \mu\right)=\mu \lambda$ for all $\left.\lambda, \mu \in \mathcal{L}\right)$. Conjugation with $\omega$ implements the squared antipode in the Hopf algebra $\mathfrak{D H}$.

Let $\mathcal{A}$ be an associative algebra and let $(\lambda, \rho)$ be a pair of homomorphisms from $\mathcal{H}$ to $\mathcal{A}$. Denote by $(\mathcal{A} \otimes \mathcal{L})_{\ell, \rho}$ the subset of elements $a=a_{1} \otimes a_{\mathcal{L}} \in \mathcal{A} \otimes \mathcal{L}$ satisfying

$$
\ell\left(h^{(1)}\right) a_{1} \otimes h^{(2)} \triangleright a_{\mathcal{L}}=a_{1} \rho(h) \otimes a_{\mathcal{L}}, \quad \forall h \in \mathcal{H} .
$$

Equivalently, condition (42) can be represented as

$$
\ell(h) a_{1} \otimes a_{\mathcal{L}}=a_{1} \rho\left(h^{(1)}\right) \otimes \bar{\gamma}\left(h^{(2)}\right) \triangleright a_{\mathcal{L}}, \quad h \in \mathcal{H} .
$$

It follows that

$$
a_{1} \rho\left(\Theta_{2}\right) \otimes \bar{\gamma}\left(\Theta_{1}\right) \triangleright a_{\mathcal{L}}=\ell\left(\bar{\Theta}_{2}\right) a_{1} \otimes\left(\bar{\omega} \bar{\Theta}_{1}\right) \triangleright a_{\mathcal{L}}
$$

for all $a=a_{1} \otimes a_{\mathcal{L}} \in(\mathcal{A} \otimes \mathcal{L})_{\ell, \rho}$. Indeed, we have for the right-hand side of (44)

$$
\begin{aligned}
\ell\left(\bar{\Theta}_{2}\right) a_{1} \otimes\left(\bar{\omega} \bar{\Theta}_{1}\right) \triangleright a_{\mathcal{L}} & =a_{1} \rho\left(\bar{\Theta}_{2}^{(1)}\right) \otimes\left(\bar{\omega} \bar{\Theta}_{1} \bar{\gamma}\left(\bar{\Theta}_{2}^{(2)}\right)\right) \triangleright a_{\mathcal{L}} \\
& =a_{1} \rho\left(\bar{\Theta}_{2}\right) \otimes\left(\bar{\omega} \bar{\Theta}_{1} \bar{\Theta}_{1^{\prime}} \bar{\gamma}\left(\bar{\Theta}_{2^{\prime}}\right)\right) \triangleright a_{\mathcal{L}}=a_{1} \rho\left(\bar{\Theta}_{2}\right) \otimes\left(\bar{\omega} \bar{\Theta}_{1} \omega\right) \triangleright a_{\mathcal{L}} .
\end{aligned}
$$

In the first equality of this chain we used (43). It remains to resort to the properties of $\omega$, $\gamma$, and $\Theta$ and get the left-hand side of(44).

Lemma 5.3. For all $a=a_{1} \otimes a_{\mathcal{L}} \in(\mathcal{A} \otimes \mathcal{L})_{\ell, \rho}$ and for any $\mu \in \mathcal{L}$ :

$$
\ell\left(\bar{\Theta}_{2}\right) a_{1} \otimes \bar{\Theta}_{1} \triangleright\left(a_{\mathcal{L}} \mu\right)=\ell\left(\bar{\Theta}_{2^{\prime}}\right) a_{1} \rho\left(\bar{\Theta}_{2}\right) \otimes\left(\bar{\Theta}_{1} \triangleright \mu\right)\left(\bar{\Theta}_{1^{\prime}} \triangleright a_{\mathcal{L}}\right) .
$$

Proof. Condition (45) is equivalent to

$$
\ell\left(\bar{\Theta}_{2}\right) a_{1} \otimes\left(\bar{\omega} \bar{\Theta}_{1}\right) \triangleright\left(a_{\mathcal{L}} \mu\right)=\ell\left(\bar{\Theta}_{2^{\prime}}\right) a_{1} \rho\left(\bar{\Theta}_{2}\right) \otimes\left(\left(\bar{\omega} \bar{\Theta}_{1}\right) \triangleright \mu\right)\left(\left(\bar{\omega} \bar{\Theta}_{1^{\prime}}\right) \triangleright a_{\mathcal{L}}\right)
$$

because $\omega$ implements a homomorphism of $\mathcal{L}$. So let us prove (46) instead of (45).

The proof employs the standard machinery of quasitriangular Hopf algebras as specialized to the double $\mathfrak{D H}$. In view of (43) and (44), the left-hand side of (46) can be transformed as

$$
\begin{aligned}
\ell\left(\bar{\Theta}_{2} \bar{\Theta}_{2^{\prime}}\right) a_{1} & \otimes\left(\left(\bar{\omega} \bar{\Theta}_{1^{\prime}}\right) \triangleright a_{\mathcal{L}}\right)\left(\left(\bar{\omega} \bar{\Theta}_{1}\right) \triangleright \mu\right)=\ell\left(\bar{\Theta}_{2}\right) a_{1} \rho\left(\Theta_{2^{\prime}}\right) \otimes\left(\left(\bar{\gamma}\left(\Theta_{1^{\prime}}\right) \triangleright a_{\mathcal{L}}\right)\left(\left(\bar{\omega} \bar{\Theta}_{1}\right) \triangleright \mu\right)\right. \\
& =a_{1} \rho\left(\bar{\Theta}_{2}^{(1)} \Theta_{2^{\prime}}\right) \otimes\left(\left(\bar{\gamma}\left(\Theta_{1^{\prime}}\right) \bar{\gamma}\left(\bar{\Theta}_{2}^{(2)}\right) \triangleright a_{\mathcal{L}}\right)\left(\left(\bar{\omega} \bar{\Theta}_{1}\right) \triangleright \mu\right)\right. \\
& =a_{1} \rho\left(\Theta_{2^{\prime}} \bar{\Theta}_{2}\right) \otimes\left(\bar{\gamma}\left(\bar{\Theta}_{2^{\prime \prime}}\right) \bar{\gamma}\left(\Theta_{1^{\prime}}\right) \triangleright a_{\mathcal{L}}\right)\left(\left(\bar{\omega} \bar{\Theta}_{1^{\prime \prime}} \bar{\Theta}_{1}\right) \triangleright \mu\right) .
\end{aligned}
$$


The last equality is the result of permutation of $\Theta$ with the coproduct and the subsequent factorization of $($ id $\otimes \Delta)(\Theta)$. Now we pull $\bar{\omega}$ through $\bar{\Theta}_{1^{\prime \prime}}$ to the right and use the $\gamma$ symmetry of $\Theta$. After that we employ $\Theta$-commutativity of the base algebra $\mathcal{L}$ and bring (477) to $a_{1} \rho\left(\Theta_{2^{\prime}} \bar{\Theta}_{2}\right) \otimes\left(\left(\bar{\omega} \bar{\Theta}_{1}\right) \triangleright \mu\right)\left(\gamma^{-1}\left(\Theta_{1^{\prime}}\right) \triangleright a_{\mathcal{L}}\right)$. Now, to get the right-hand side of (46), we again apply (44) in the reversed direction.

\subsection{An anti-automorphism of $\check{\mathcal{U}}_{\text {sym }}^{n}$}

In this subsection we introduce an important family of endomorphisms of dynamical tensors. This family yields a kind of anti-automorphism of the "algebra" of symmetric dynamical tensors.

Introduce a linear operator $\Gamma \in \operatorname{Aut}(\check{\mathcal{U}})$ setting

$$
\Gamma(x \otimes \lambda):=\gamma\left(\bar{\Theta}_{2} x\right) \otimes \bar{\Theta}_{1} \triangleright \lambda
$$

for all $x \otimes \lambda \in \check{\mathcal{U}}$. Further, define a linear automorphism $\Gamma_{i}$ of $\check{\mathcal{U}}^{n}$ as $\Gamma$ acting on the i-th $\mathcal{U}$ and the $\mathcal{L}$-factor of $\check{\mathcal{U}}^{n}$. The operators $\Gamma_{i}$ and $\Gamma_{j}$ do not commute unless $i=j$. Define a linear automorphism $\Gamma_{1 \ldots n} \in \operatorname{Aut}\left(\check{\mathcal{U}}^{n}\right)$ for $n \in \mathbb{N}$ setting $\Gamma_{1 \ldots n}:=\Gamma_{1} \ldots \Gamma_{n}$. Put also $\Gamma_{\sigma}:=\sigma \circ \Gamma_{1 \ldots n}$ for $\sigma \in \mathfrak{S}_{n}$; then the operator $\Gamma_{\sigma}$ can be represented as

$$
\Gamma_{\sigma}\left(x_{1 \ldots n} \otimes x_{\mathcal{L}}\right)=\gamma^{\otimes n}\left(\Delta_{\sigma}^{n}\left(\bar{\Theta}_{2}\right) x_{1 \ldots n}\right) \otimes \bar{\Theta}_{1} \triangleright x_{\mathcal{L}} \quad \text { for } x=x_{1 \ldots n} \otimes x_{\mathcal{L}} \in \mathcal{U}^{\otimes n} \otimes \mathcal{L}=\check{\mathcal{U}}^{n}
$$

Similarly we define the operators $\bar{\Gamma}_{1 \ldots n}:=\bar{\Gamma}_{1} \ldots \bar{\Gamma}_{n}$ and $\bar{\Gamma}_{\sigma}:=\sigma \circ \bar{\Gamma}_{1 \ldots n}$ for $\sigma \in \mathfrak{S}_{n}$. We have $\left(\Gamma_{\sigma}\right)^{-1}=\bar{\Gamma}_{\sigma^{\prime}}$, where the map $\alpha \mapsto \alpha^{\prime}$ is the inner automorphism of $\mathfrak{S}_{n}$ with the element $\tau$, namely $\alpha^{\prime}=\tau \alpha \bar{\tau}$.

The collection $\left\{\Gamma_{\alpha}\right\}_{\alpha \in \mathfrak{S}_{n}}$ is an anti-homomorphism of the algebra of symmetric tensors. Moreover, it implements an isomorphism between the left and right $\check{\mathcal{U}}_{\text {sym }}^{n}$-" module structure" on $\check{\mathcal{U}}^{n}$. To be precise, we have the following proposition.

Proposition 5.4. (i) Fix a pair of permutations $\alpha, \beta \in \mathfrak{S}_{n}$. Then

$$
\begin{gathered}
\Gamma_{\alpha}\left({ }_{\alpha} \check{\mathcal{U}}_{\beta}{ }^{n}\right) \subset{ }_{\beta^{\prime}} \check{\mathcal{U}}_{\alpha^{\prime}}{ }^{n}, \quad \bar{\Gamma}_{\alpha}\left({ }_{\alpha} \check{\mathcal{U}}_{\beta}{ }^{n}\right) \subset{ }_{\beta} \check{\mathcal{U}}_{\alpha^{\prime}}{ }^{n} \quad \text { and } \\
\Gamma_{\alpha}(x y)=\Gamma_{\beta}(y) \Gamma_{\alpha}(x), \quad \bar{\Gamma}_{\alpha}(y x)=\bar{\Gamma}_{\alpha}(x) \bar{\Gamma}_{\beta}(y)
\end{gathered}
$$

for all $x \in{ }_{\alpha} \check{\mathcal{U}}_{\beta}^{n}$ and $y \in \check{\mathcal{U}}^{n}$.

(ii) For all $x \in \check{\mathcal{U}}$ one has

$$
\left(\Gamma_{\sigma} \circ \Delta_{\sigma}^{n}\right)(x)=\left(\Delta_{\sigma^{\prime}}^{n} \circ \Gamma\right)(x), \quad\left(\bar{\Gamma}_{\sigma} \circ \Delta_{\sigma}^{n}\right)(x)=\left(\Delta_{\sigma^{\prime}}^{n} \circ \bar{\Gamma}\right)(x),
$$


Proof. The right formulas in (48/50) follow from the left ones, as $\left(\Gamma_{\sigma}\right)^{-1}=\bar{\Gamma}_{\sigma^{\prime}}$. Let us sketch the proof for the left ones. The left inclusion (48) is a corollary of (47) and $\left(\Gamma_{\sigma}\right)^{-1}=\bar{\Gamma}_{\sigma^{\prime}}$. The left equality (49) follows from Lemma 5.3. These prove (i). Statement (ii) follows from (477). The details are left to the reader.

Example 5.5. As an application of Proposition 5.4, apply $\Gamma_{321}$ to the dYBE (41), which is an equation in $\check{U}_{\text {sym }}^{3}$. It is easy to see that $\Gamma_{321}$ implements an automorphism of (41), and the correspondence $\tilde{\mathcal{R}} \mapsto \Gamma_{21}(\tilde{\mathcal{R}})$ maps a solution to another solution.

\section{A gauge transformation of dynamical twist}

In the theory of Hopf algebras, a twist transformation changes the comultiplication and the antipode by a similarity transformation. The elements $\vartheta$ and $\bar{\vartheta}=\vartheta^{-1}$ defining the new antipode $\tilde{\gamma}(h)=\bar{\vartheta}^{-1} \gamma(h) \vartheta$ are expressed through the twisting cocycle $\mathcal{F}$ as $\vartheta=\gamma\left(\mathcal{F}_{1}\right) \mathcal{F}_{2}$ and $\bar{\vartheta}=\overline{\mathcal{F}}_{1} \gamma\left(\overline{\mathcal{F}}_{2}\right)$. They implement the gauge-equivalence between the twists $\mathcal{F}$ and $(\gamma \otimes \gamma)\left(\overline{\mathcal{F}}_{21}\right)$, see [Dr3]:

$$
\Delta(\vartheta) \mathcal{F}=(\gamma \otimes \gamma)\left(\overline{\mathcal{F}}_{21}\right)(\vartheta \otimes \vartheta)
$$

It turns out that the dynamical version of the element $\vartheta$, as well as the equation (51), does exist. They play an important role in our consideration.

\subsection{Elements $\vartheta$ and $\zeta$}

Let $\mathcal{F}=\mathcal{F}_{1} \otimes \mathcal{F}_{2} \otimes \mathcal{F}_{\mathcal{L}} \in{ }_{\epsilon} \check{\mathcal{U}}_{\epsilon}^{2}$ be a dynamical twisting cocycle. Introduce the elements $\vartheta, \zeta \in \mathcal{U} \otimes \mathcal{L}$ by

$$
\vartheta:=\gamma\left(\mathcal{F}_{1}\right) \mathcal{F}_{2} \otimes \mathcal{F}_{\mathcal{L}}=\vartheta_{1} \otimes \vartheta_{\mathcal{L}}, \quad \zeta:=\overline{\mathcal{F}}_{2} \bar{\gamma}\left(\overline{\mathcal{F}}_{1}\right) \otimes \overline{\mathcal{F}}_{\mathcal{L}}=\zeta_{1} \otimes \zeta_{\mathcal{L}}
$$

Since $\mathcal{F} \in \check{\mathcal{U}}_{\epsilon}^{2}$, one can show that $\vartheta$ and $\zeta$ belong to $\check{\mathcal{U}}_{\epsilon}$.

Lemma 6.1. Let $\mathcal{F}$ be a dynamical twisting cocycle and $\vartheta, \zeta$ defined by (5q). Then

$$
\begin{aligned}
& \mathcal{F}_{1}^{(1)} \otimes \gamma\left(\mathcal{F}_{1}^{(2)}\right) \mathcal{F}_{2} \otimes \mathcal{F}_{\mathcal{L}}=\Gamma_{2}(\overline{\mathcal{F}}) \vartheta_{2}, \\
& \gamma\left(\mathcal{F}_{1}\right) \mathcal{F}_{2}^{(1)} \otimes \mathcal{F}_{2}^{(2)} \otimes \mathcal{F}_{\mathcal{L}}=\vartheta_{1 \mid 2} \overline{\mathcal{F}}, \\
& \overline{\mathcal{F}}_{2}^{(1)} \bar{\gamma}\left(\overline{\mathcal{F}}_{1}\right) \otimes \overline{\mathcal{F}}_{2}^{(2)} \otimes \overline{\mathcal{F}}_{\mathcal{L}}=\mathcal{F} \zeta_{1 \mid 2}, \\
& \overline{\mathcal{F}}_{1}^{(1)} \otimes \overline{\mathcal{F}}_{2} \bar{\gamma}\left(\overline{\mathcal{F}}_{1}^{(2)}\right) \otimes \overline{\mathcal{F}}_{\mathcal{L}}=\zeta_{2} \bar{\Gamma}_{2}(\mathcal{F}) .
\end{aligned}
$$


Proof. Direct consequence of the conditions (39) and (401).

Proposition 6.2. The elements $\vartheta$ and $\zeta$ are invertible and

$$
\bar{\vartheta}=\Gamma(\zeta), \quad \bar{\zeta}=\bar{\Gamma}(\vartheta)
$$

Proof. Note that one of these equations implies the other since $\Gamma$ is an anti-automorphism of $\check{\mathcal{U}}_{\epsilon}$.

We will deduce the left equality (57) from (53) and (56). First observe that taking product of the $\mathcal{U}$-factors in $\Gamma_{2}(\overline{\mathcal{F}})$ gives $\Gamma(\zeta)$. Then (53) implies $1_{\breve{\mathcal{U}}}=\Gamma(\zeta) \vartheta$, i.e. $\Gamma(\zeta)$ is a left inverse to $\vartheta$. From (56) we obtain $1_{\breve{U}}=\zeta \bar{\Gamma}(\vartheta)$. Apply to this equation the operator $\Gamma$, which is an anti-algebra automorphism of $\check{\mathcal{U}}_{\epsilon}$. This shows that $\Gamma(\zeta)$ is also a right inverse to $\vartheta$.

\subsection{The gauge transformation}

A dynamical twist induces an isomorphic transformation of the dynamical extension of the monoidal category of $\mathcal{U}$-modules, DM1. Therefore it can be composed with a coboundary twist (a coboundary twist may be associated with an arbitrary collection of automorphisms). We call such coboundary twist a gauge transformation. A gauge transformation of a dynamical twist $\mathcal{F}$ is defined by any invertible element $\xi \in{ }_{\epsilon} \check{\mathcal{U}}_{\epsilon}$ and reads

$$
\mathcal{F} \mapsto \Delta(\bar{\xi}) \mathcal{F} \xi_{1 \mid 2} \xi_{2}
$$

Proposition 6.3. The symmetric dynamical tensor $\left(\Gamma_{21}\right)\left(\overline{\mathcal{F}}_{21}\right) \in \check{\epsilon}_{\epsilon}{ }^{2}$ is gauge-equivalent to $\mathcal{F}$ with

$$
\begin{aligned}
\Delta(\vartheta) \mathcal{F} & =\left(\Gamma_{21}\right)\left(\overline{\mathcal{F}}_{21}\right)\left(\vartheta_{1 \mid 2} \vartheta_{2}\right) \\
\overline{\mathcal{F}} \Delta(\zeta) & =\left(\zeta_{1 \mid 2} \zeta_{2}\right)\left(\bar{\Gamma}_{21}\right)\left(\mathcal{F}_{21}\right)
\end{aligned}
$$

Proof. Let us compute the expression $\Delta(\vartheta) \mathcal{F}$. Using (39), we find

$$
\Delta(\vartheta) \mathcal{F}=\left(\Delta\left(\gamma\left(\mathcal{F}_{1}\right)\right) \Delta\left(\mathcal{F}_{2}\right) \otimes \mathcal{F}_{\mathcal{L}}\right) \mathcal{F}=\Delta\left(\gamma\left(\mathcal{F}_{1}^{(1)} \mathcal{F}_{1^{\prime}}\right)\right)\left(\mathcal{F}_{1}^{(2)} \mathcal{F}_{2^{\prime}} \otimes \mathcal{F}_{2} \mathcal{F}_{\mathcal{L}^{\prime}}^{(1)}\right) \otimes \mathcal{F}_{\mathcal{L}} \mathcal{F}_{\mathcal{L}^{\prime}}^{[\infty]}
$$

Permuting the coproduct and antipode and using (40), we find this equal to

$$
\gamma\left(\mathcal{F}_{1^{\prime}}^{(2)}\right) \mathcal{F}_{2^{\prime}} \otimes \gamma\left(\mathcal{F}_{1^{\prime}}^{(1)}\right) \gamma\left(\mathcal{F}_{1}\right) \mathcal{F}_{2} \mathcal{F}_{\mathcal{L}^{\prime}}^{(1)} \otimes \mathcal{F}_{\mathcal{L}} \mathcal{F}_{\mathcal{L}^{\prime}}^{[\infty]}=\left(\gamma\left(\mathcal{F}_{1^{\prime}}^{(2)}\right) \mathcal{F}_{2^{\prime}} \otimes \gamma\left(\mathcal{F}_{1^{\prime}}^{(1)}\right) \mathcal{F}_{\mathcal{L}^{\prime}}^{(1)} \otimes \mathcal{F}_{\mathcal{L}^{\prime}}^{[\infty]}\right) \vartheta_{2}
$$


Here we used the fact that $\vartheta$ belongs to ${ }_{\epsilon} \check{\mathcal{U}}_{\epsilon}$ and therefore commutes with $\delta\left(\mathcal{F}_{\mathcal{L}^{\prime}}\right)$. Now let us compute left factor in the right-hand side of (601). From (53) we deduce

$$
\gamma\left(\mathcal{F}_{1}^{(2)}\right) \mathcal{F}_{2} \otimes \gamma\left(\mathcal{F}_{1}^{(1)}\right) \mathcal{F}_{\mathcal{L}}^{(1)} \otimes \mathcal{F}_{\mathcal{L}}^{[\infty]}=\Gamma_{2}\left(\Gamma_{1}\left(\overline{\mathcal{F}}_{21}\right) \vartheta_{1}\right)=\Gamma_{21}\left(\overline{\mathcal{F}}_{21}\right) \vartheta_{1 \mid 2}
$$

The last equality can be checked directly. Substituting this into (60), we prove the formula (58).

Now we derive (59) from (58). First of all observe, using the right equality (57) and Proposition 5.4, that

$$
\begin{aligned}
\left(\bar{\Gamma}_{12} \circ \Delta\right)(\vartheta) & =\left(\Delta_{\tau} \circ \bar{\Gamma}\right)(\vartheta)=\Delta_{\tau}(\bar{\zeta}), \\
\left(\bar{\Gamma}_{12}\right)\left(\vartheta_{1 \mid 2} \vartheta_{2}\right) & =\left(\bar{\Gamma}_{1} \bar{\Gamma}_{2}\right)\left(\vartheta_{2}\right)\left(\bar{\Gamma}_{1} \bar{\Gamma}_{2}\right)\left(\vartheta_{1 \mid 2}\right)=\bar{\zeta}_{2 \mid 1} \bar{\zeta}_{1}
\end{aligned}
$$

Note that $\Delta_{\tau}=\Delta_{o p}$. Here we employed the fact that $\vartheta_{1 \mid 2}$ and $\vartheta_{2}$ belong to $\check{\mathcal{U}}_{\epsilon}^{2}$. Applying $\tau \circ \bar{\Gamma}_{12}$ to (58) and using the above equalities, we obtain (59).

\subsection{Symmetries of dynamical R-matrix}

In the theory of quasitriangular Hopf algebras, the universal R-matrices transform under the action of antipode as

$$
(\gamma \otimes \gamma)(\mathcal{R})=\mathcal{R}, \quad(\gamma \otimes \mathrm{id})(\mathcal{R})=\overline{\mathcal{R}}, \quad(\mathrm{id} \otimes \gamma)(\overline{\mathcal{R}})=\mathcal{R}
$$

In the present subsection we establish dynamical analogs of these relations.

Proposition 6.4. Suppose that $\mathcal{U}$ is a quasitriangular Hopf algebra with the universal $R$ matrix $\mathcal{R}$. Let $\mathcal{F}$ be a dynamical $(\mathcal{U}, \mathcal{H}, \mathcal{L})$-twist, $\tilde{\mathcal{R}}=\mathcal{F}_{21}^{-1} \mathcal{R} \mathcal{F}$ the corresponding dynamical $R$-matrix, and $\zeta$ defined as in (52). Then

$$
\begin{aligned}
\left(\Gamma_{2} \Gamma_{1}\right)\left(\bar{\zeta}_{2 \mid 1} \bar{\zeta}_{1} \tilde{\mathcal{R}} \zeta_{1 \mid 2} \zeta_{2}\right) & =\tilde{\mathcal{R}}=\left(\bar{\Gamma}_{1} \bar{\Gamma}_{2}\right)\left(\vartheta_{2 \mid 1} \vartheta_{1} \tilde{\mathcal{R}} \bar{\vartheta}_{1 \mid 2} \bar{\vartheta}_{2}\right) \\
\Gamma_{1}\left(\bar{\zeta}_{1} \tilde{\mathcal{R}} \zeta_{1 \mid 2}\right) & =\tilde{\mathcal{R}}^{-1}=\bar{\Gamma}_{2}\left(\vartheta_{2 \mid 1} \tilde{\mathcal{R}}_{2}\right) \\
\Gamma_{2}\left(\bar{\zeta}_{2} \tilde{\mathcal{R}}^{-1} \zeta_{2 \mid 1}\right) & =\tilde{\mathcal{R}}=\bar{\Gamma}_{1}\left(\vartheta_{1 \mid 2} \tilde{\mathcal{R}}^{-1} \bar{\vartheta}_{1}\right) .
\end{aligned}
$$

Proof. Let us establish the left equality (62) first. Using (55) we find

$$
\overline{\mathcal{F}}_{21} \mathcal{R} \mathcal{F} \zeta_{1 \mid 2}=\overline{\mathcal{F}}_{2^{\prime}} \mathcal{R}_{1}\left(\overline{\mathcal{F}}_{2}^{(1)} \bar{\gamma}\left(\overline{\mathcal{F}}_{1}\right)\right) \otimes \overline{\mathcal{F}}_{1^{\prime}} \mathcal{R}_{2}\left(\overline{\mathcal{F}}_{2}^{(2)}\right) \otimes \overline{\mathcal{F}}_{\mathcal{L}^{\prime}}\left(\overline{\mathcal{F}}_{\mathcal{L}}\right)
$$

Pulling the term $\Delta\left(\mathcal{F}_{2}\right)$ through the R-matrix to the left and then applying the dynamical cocycle condition (39) we transform the right-hand side of (64) to

$\overline{\mathcal{F}}_{\mathcal{L}^{\prime}}^{(1)} \overline{\mathcal{F}}_{2} \mathcal{R}_{1} \bar{\gamma}\left(\overline{\mathcal{F}}_{1^{\prime}} \overline{\mathcal{F}}_{1}^{(1)}\right) \otimes \overline{\mathcal{F}}_{2^{\prime}} \overline{\mathcal{F}}_{1}^{(2)} \mathcal{R}_{2} \otimes \overline{\mathcal{F}}_{\mathcal{L}^{\prime}}^{[\infty]} \overline{\mathcal{F}}_{\mathcal{L}}=\overline{\mathcal{F}}_{\mathcal{L}^{\prime}}^{(1)} \overline{\mathcal{F}}_{2} \bar{\gamma}\left(\overline{\mathcal{F}}_{1}^{(2)}\right) \mathcal{R}_{1} \bar{\gamma}\left(\overline{\mathcal{F}}_{1^{\prime}}\right) \otimes \overline{\mathcal{F}}_{2^{\prime}} \mathcal{R}_{2} \overline{\mathcal{F}}_{1}^{(1)} \otimes \overline{\mathcal{F}}_{\mathcal{L}^{\prime}}^{[\infty]} \overline{\mathcal{F}}_{\mathcal{L}}$ 
where we used the equality $\mathcal{R}_{1} \bar{\gamma}\left(\mathcal{F}_{1}^{(1)}\right) \otimes \mathcal{F}_{1}^{(2)} \mathcal{R}_{2}=\bar{\gamma}\left(\mathcal{F}_{1}^{(2)}\right) \mathcal{R}_{1} \otimes \mathcal{R}_{2} \mathcal{F}_{1}^{(2)}$. Now we appeal to (56)) and find (64) equal to $\overline{\mathcal{F}}_{\mathcal{L}^{\prime}}^{(1)} \zeta_{1} \mathcal{F}_{\mathcal{L}^{\prime \prime}}^{(1)} \bar{\gamma}\left(\mathcal{F}_{2^{\prime \prime}}\right) \mathcal{R}_{1} \bar{\gamma}\left(\overline{\mathcal{F}}_{1^{\prime}}\right) \otimes \overline{\mathcal{F}}_{2^{\prime}} \mathcal{R}_{2} \mathcal{F}_{1^{\prime \prime}} \otimes \overline{\mathcal{F}}_{\mathcal{L}^{\prime}}^{[\infty]} \zeta_{\mathcal{L}} \mathcal{F}_{\mathcal{L}^{\prime \prime}}^{[\infty]}$. Since $\zeta \in \check{\mathcal{U}}_{\epsilon}$, it commutes with $\delta(\mathcal{L})$ and can be pulled to the left. Thus we come to

$$
\begin{aligned}
\overline{\mathcal{F}}_{21} \mathcal{R} \mathcal{F} \zeta_{1 \mid 2} & =\zeta_{1} \overline{\mathcal{F}}_{\mathcal{L}^{\prime}}^{(1)} \mathcal{F}_{\mathcal{L}^{\prime \prime}}^{(1)} \bar{\gamma}\left(\mathcal{F}_{2^{\prime \prime}}\right) \mathcal{R}_{1} \bar{\gamma}\left(\overline{\mathcal{F}}_{1^{\prime}}\right) \otimes \overline{\mathcal{F}}_{2^{\prime}} \mathcal{R}_{2} \mathcal{F}_{1^{\prime \prime}} \otimes \zeta_{\mathcal{L}} \overline{\mathcal{F}}_{\mathcal{L}^{\prime}}^{[\infty]} \mathcal{F}_{\mathcal{L}^{\prime \prime}}^{[\infty]} \\
& =\zeta_{1}\left(\Theta_{2} \bar{\gamma}\left(\tilde{\mathcal{R}}_{1}\right) \otimes \tilde{\mathcal{R}}_{2} \otimes \Theta_{1} \triangleright \tilde{\mathcal{R}}_{\mathcal{L}}\right) .
\end{aligned}
$$

This immediately implies the left equality (62).

Further, using (59), we find

$$
\bar{\zeta}_{2 \mid 1} \bar{\zeta}_{1} \tilde{\mathcal{R}} \zeta_{1 \mid 2} \zeta_{2}=\bar{\Gamma}_{12}(\mathcal{F}) \mathcal{R} \bar{\Gamma}_{21}\left(\overline{\mathcal{F}}_{21}\right)
$$

Observe that each factor on the right-hand side has definite symmetry type, and this product has the structure ${ }_{\tau} \check{\mathcal{U}}_{\tau}{ }^{2}{ }_{\tau} \check{\mathcal{U}}_{\epsilon}{ }_{\epsilon} \check{\mathcal{U}}_{\epsilon}{ }^{2}$. Apply to this equation the map $\Gamma_{21}=\Gamma_{2} \Gamma_{1}=\left(\bar{\Gamma}_{12}\right)^{-1}$ and use Proposition 5.4 (i), formula (49). This proves the left equality (61), from which we deduce the right one. Indeed, the left equality (61) implies

$$
\tilde{\mathcal{R}}=\left(\Gamma_{1} \Gamma_{2}\right)\left(\zeta_{1 \mid 2} \zeta_{2}\right)\left(\Gamma_{2} \Gamma_{1}\right)(\tilde{\mathcal{R}})\left(\Gamma_{2} \Gamma_{1}\right)\left(\bar{\zeta}_{2 \mid 1} \bar{\zeta}_{1}\right)
$$

Here we again used Proposition 5.4 (i), formula (49). It remains to apply formulas (57), to get the right equality (61).

The right equality (62) follows from (61) and the left equality (62). Indeed, observe that the element $\bar{\zeta}_{1} \tilde{\mathcal{R}} \zeta_{1 \mid 2}$ belongs to ${ }_{\tau} \check{\mathcal{U}}_{\epsilon}^{2}$. Employing (49) we find

$$
\tilde{\mathcal{R}}=\left(\Gamma_{2} \Gamma_{1}\right)\left(\bar{\zeta}_{2 \mid 1} \bar{\zeta}_{1} \tilde{\mathcal{R}} \zeta_{1 \mid 2} \zeta_{2}\right)=\left(\Gamma_{1} \Gamma_{2}\right)\left(\zeta_{2}\right)\left(\Gamma_{2} \Gamma_{1}\right)\left(\bar{\zeta}_{1} \tilde{\mathcal{R}}_{1 \mid 2}\right)\left(\Gamma_{2} \Gamma_{1}\right)\left(\bar{\zeta}_{2 \mid 1}\right)=\bar{\vartheta}_{2 \mid 1} \Gamma_{2}\left(\tilde{\mathcal{R}}^{-1}\right) \vartheta_{2} .
$$

The last equality is obtained using (57) and the left equality (62).

The left and right equalities (63) are corollaries of the right and left equalities (62) respectively. This completes the proof.

Remark 6.5. The gauge equivalence (58)-(58) as well as the formulas (61)- (63) take place because of rigidity of the dynamical category of finite dimensional $\mathcal{H}$-modules. Indeed, the dynamical category is rigid, being an extension of the rigid category of finite dimensional $\mathcal{H}$-modules by enlarging homsets, see [DM1].

\section{Dynamical twist of the RE algebra}

\subsection{Dynamical twist and quantum groupoids}

All the bialgebroids in this subsection are assumed to be left. Here we recall the connection between the dynamical twist and twist of bialgebroids following [Xu1, DM2]. 
Given a Hopf algebra inclusion $\mathcal{H} \subset \mathcal{U}$ and a dynamical $(\mathcal{U}, \mathcal{H}, \mathcal{L})$-twist $\mathcal{F}$, it is possible to construct a twisted left $\mathcal{L}$-bialgebroid $\mathcal{U} \widetilde{\otimes \mathfrak{D H}} \mathcal{L}_{\mathcal{L}}$ starting from the tensor product $\mathcal{L}$ bialgebroid $\mathcal{U} \otimes \mathfrak{D H}_{\mathcal{L}}$, see DM2]. This latter bialgebroid is obtained by a projection from $\mathcal{U} \otimes \mathcal{L} \rtimes \mathfrak{D H}$, cf. Example 2.6. We will use this projection representation for elements of $\mathcal{U} \otimes \mathfrak{D H}_{\mathcal{L}}$. Under this convention, the bialgebroid twist $\Psi \in\left(\mathcal{U} \otimes \mathfrak{D H} \mathcal{H}_{\mathcal{L}}\right) \otimes_{\mathcal{L}}\left(\mathcal{U} \otimes \mathfrak{D H} \mathcal{H}_{\mathcal{L}}\right)$ is expressed through $\mathcal{F}$ and $\Theta$ by

$$
\Psi:=\left(\mathcal{F}_{1} \otimes \mathcal{F}_{3} \otimes \Theta_{1}\right) \otimes_{\mathcal{L}}\left(\mathcal{F}_{2} \Theta_{2} \otimes 1_{\mathcal{L}} \otimes 1_{\mathfrak{D H}}\right)
$$

We assume that $\mathcal{U}$ is a quasitriangular Hopf algebra with the universal R-matrix $\mathcal{R}$. Then the bialgebroids $\mathcal{U} \otimes \mathfrak{D H} \mathcal{H}_{\mathcal{L}}$ and $\mathcal{U} \widetilde{\mathcal{D H}} \mathcal{H}_{\mathcal{L}}$ are also quasitriangular, [DM2]. The universal R-matrix of $\widetilde{\mathcal{U}}_{\mathbb{D} \mathcal{H}_{\mathcal{L}}}$ reads

$$
\mathbf{R}=\left(\bar{\Theta}_{2^{\prime}} \bar{\Theta}_{2^{\prime \prime \prime}} \tilde{\mathcal{R}}_{1} \otimes 1_{\mathcal{L}} \otimes \Theta_{1} \Theta_{1^{\prime \prime}}\right) \otimes_{\mathcal{L}_{o p}}\left(\tilde{\mathcal{R}}_{2} \Theta_{2^{\prime \prime}} \otimes \bar{\Theta}_{1^{\prime \prime \prime}} \triangleright \tilde{\mathcal{R}}_{3} \otimes \bar{\Theta}_{1^{\prime}} \Theta_{2}\right),
$$

where $\tilde{\mathcal{R}}=\overline{\mathcal{F}}_{21} \mathcal{R} \mathcal{F}=\tilde{\mathcal{R}}_{1} \otimes \tilde{\mathcal{R}}_{2} \otimes \tilde{\mathcal{R}}_{3} \in \mathcal{U} \otimes \mathcal{U} \otimes \mathcal{L}$ is the dynamical R-matrix, the twist of $\mathcal{R}$.

Let $\mathcal{A}$ be a left $\mathcal{U}$-module algebra and $\checkmark$ denote the $\mathcal{U}$-action on $\mathcal{A}$. Recall that $\mathcal{L}$ is a module algebra over the bialgebroid $\mathfrak{D} \mathcal{H}_{\mathcal{L}}$ with respect to the anchor action. Then the tensor product $\mathcal{A} \otimes \mathcal{L}$ is a natural module (and module algebra) over the tensor product bialgebroid $\mathcal{U} \otimes \mathfrak{D H} \mathcal{H}_{\mathcal{L}}$. Recall from Example 2.6 that $\mathfrak{D H}$ is a quotient of $\mathcal{L} \rtimes \mathfrak{D H}$. The action of $\mathcal{U} \otimes \mathfrak{D H} \mathcal{L}$ is induced by the action of $\mathcal{U} \otimes \mathcal{L} \rtimes \mathfrak{D H}$, which is given by

$$
(u \otimes \lambda \otimes h) \otimes(a \otimes \mu) \mapsto u \triangleright a \otimes \lambda(h \triangleright \mu),
$$

for $(u \otimes \lambda \otimes h) \in \mathcal{U} \otimes \mathcal{L} \rtimes \mathfrak{D H}$ and $a \otimes \mu \in \mathcal{A} \otimes \mathcal{L}$.

Suppose that $\mathcal{A}$ is a commutative algebra in the braided category of $\mathcal{U}$-modules, i.e.

$$
\left(\mathcal{R}_{2} \triangleright b\right)\left(\mathcal{R}_{1} \triangleright a\right)=a b, \quad \text { for all } \quad a, b \in \mathcal{A} \text {. }
$$

Then $\mathcal{A} \otimes \mathcal{L}$ is a commutative algebra in the braided category of $\mathcal{U} \otimes \mathfrak{D H} \mathcal{H}_{\mathcal{L}}$-modules. The bialgebroid twist defines an automorphism of the category of $\mathcal{U} \otimes \mathfrak{D H}_{\mathcal{L}}$-modules, so it transforms a commutative algebra into commutative, with respect to the new braiding.

The bialgebroid twist that comes from a dynamical one re-defines the multiplication in the algebra $\mathcal{A} \otimes \mathcal{L}$ according to the rule

$$
\begin{aligned}
a * b & =\left(\mathcal{F}_{1} \triangleright a\right)\left(\mathcal{F}_{2} \triangleright b\right) \otimes \mathcal{F}_{\mathcal{L}}, & \lambda * \mu & =(1 \otimes \lambda \mu), \\
\lambda * a & =\lambda^{(1)} a \otimes \lambda^{[\infty]}, & a * \lambda & =a \otimes \lambda .
\end{aligned}
$$


for $a, b \in \mathcal{A} \subset \mathcal{A} \otimes \mathcal{L}$ and $\lambda, \mu \in \mathcal{L} \subset \mathcal{A} \otimes \mathcal{L}$. The new algebra $\tilde{\mathcal{A}}$ is that over the twisted quantum groupoid $\widehat{\mathcal{U}} \widehat{\otimes \mathfrak{D H}} \mathcal{L}_{\mathcal{L}}$ and, in particular, it is an $\mathcal{L}$-bimodule algebra. It is commutative with respect to the permutation induced by $\mathbf{R}$.

It is seen that the permutation rule between $\mathcal{L} \subset \tilde{\mathcal{A}}$ and the entire $\tilde{\mathcal{A}}$ is governed by $\Theta$, the R-matrix of the double $\mathfrak{D H}$. Permutation among elements from $\mathcal{A} \subset \tilde{\mathcal{A}}$ is expressed through the dynamical R-matrix by the following obvious formula.

$$
\left(\tilde{\mathcal{R}}_{2} \triangleright b\right) *\left(\tilde{\mathcal{R}}_{1} \triangleright a\right) * \tilde{\mathcal{R}}_{\mathcal{L}} * \lambda=a * b * \lambda
$$

for any $a, b \in \mathcal{A}$ and $\lambda \in \mathcal{L}$.

\section{2 dFRT algebra via dynamical twist}

Let $(V, \rho)$ be a $\mathcal{U}$-module and therefore an $\mathcal{H}$-module by restriction. Denote by $R$ and $\tilde{R}$ the images of $\mathcal{R}$ and $\tilde{\mathcal{R}}$ in this representation. Set $S=P R$ and $\tilde{S}=P \tilde{R}$, where $P$ is the standard matrix permutation on $V \otimes V$. We claim that in this case the dFRT algebra associated with $\tilde{S}$ is a dynamical twist of $\mathcal{T}$ associated with $S$.

The FRT algebra $\mathcal{T}$ is generated by the matrix coefficients $\left\|T_{j}^{i}\right\|$ of the representation $\rho$ subject to relations

$$
S T_{1} T_{2}=T_{1} T_{2} S
$$

The $\mathcal{U}$-bimodule structure $\mathcal{T}$ is defined by the formulas (15), where $x \in \mathcal{U}$ and $y \in \mathcal{U}_{\text {op }}$.

Let us consider $\mathcal{T} \otimes \mathcal{L}_{o p} \otimes \mathcal{L}$ as an algebra over the $\mathcal{L}_{o p} \otimes \mathcal{L}$-bialgebroid $\mathcal{B}_{o p} \otimes \mathcal{B}$, where $\mathcal{B}=\mathcal{U} \otimes \mathfrak{D H} \mathcal{H}_{\mathcal{L}}$ and $\mathcal{B}_{o p}$ differs from $\mathcal{B}$ by the replacement of $(\mathcal{U}, \mathcal{H}, \mathcal{L})$ by $\left(\mathcal{U}_{o p}, \mathcal{H}_{o p}, \mathcal{L}_{o p}\right)$, see DM2]. Recall from Example 2.5 that $\mathcal{L}_{o p}$ is an $\mathcal{H}_{o p}$-base algebra.

Suppose that $\mathcal{F} \in \check{\mathcal{U}}_{\epsilon}{ }^{2}$ is a universal dynamical cocycle over the base $\mathcal{L}$. Then $\overline{\mathcal{F}}:=$ $\mathcal{F}^{-1} \in \mathcal{U}_{o p} \otimes \mathcal{U}_{o p} \otimes \mathcal{L}_{o p}$ is a universal dynamical cocycle over $\mathcal{L}_{o p}$, DM2]. Thus $\overline{\mathcal{F}} \otimes \mathcal{F}$ is a universal twist in the dynamical extension of the category of $\mathcal{U}$-bimodules over the base algebra $\mathcal{L}_{o p} \otimes \mathcal{L}$. In the standard way, it amounts to a twist of the bialgebroid $\mathcal{B}_{\text {op }} \otimes \mathcal{B}$. Denote by $\tilde{\mathcal{T}}^{\prime}$ the corresponding twist of the module algebra $\mathcal{T} \otimes \mathcal{L}_{o p} \otimes \mathcal{L}$, as explained in Subsection 7.1 ,

Proposition 7.1. The algebra $\tilde{\mathcal{T}}^{\prime}$ is isomorphic to the dFRT algebra $\tilde{\mathcal{T}}$ from Definition 3.1.

Proof. Immediate corollary of (70) and (71). 
Now consider the dual Hopf algebra $\mathcal{U}^{*}$ to $\mathcal{U}$ as a bimodule over $\mathcal{U}$. This algebra is generated by the matrix coefficients of a fundamental representation of $\mathcal{U}$. They satisfy the ordinary FRT relations plus some additional relations, e.g. the unit quantum determinant in the $\mathcal{U}=\mathcal{U}_{q}(s l(n))$-case. Apply the dynamical twist $\overline{\mathcal{F}} \otimes \mathcal{F}$ to $\mathcal{U}^{*} \rtimes\left(\mathcal{L}_{o p} \otimes \mathcal{L}\right)$ and construct the algebra $\tilde{\mathcal{U}}^{*}$ similarly to $\tilde{\mathcal{T}}^{\prime} \simeq \tilde{\mathcal{T}}$ above.

Consider the matrix coefficients of the representation $\rho$ as elements of $\mathcal{U}^{*}$ arranged into the matrix $U=\left\|U_{j}^{i}\right\|_{i, j=1}^{\operatorname{dim} V}$. Observe that $U$ is invertible in $\operatorname{End}(V) \otimes \mathcal{U}^{*}$ and $U^{-1}=\gamma_{\mathcal{U}^{*}}(U)$. We are going to prove that the matrix $U$ is invertible when its entries are regarded as elements of $\tilde{\mathcal{U}}^{*}$. Introduce the matrix $\bar{U} \in \operatorname{End}(V) \otimes \tilde{\mathcal{U}}^{*}$ setting

$$
\bar{U}:=\rho\left(\gamma\left(\zeta_{1}\right)\right) \gamma_{\mathcal{U}^{*}}(U) \rho\left(\gamma\left(\bar{\zeta}_{1}\right)\right) \otimes \bar{\zeta}_{\mathcal{L}} \otimes \zeta_{\mathcal{L}} .
$$

Here $\gamma$ is the antipode in $\mathcal{U}$ and $\zeta$ is defined in (52). Note that the component $\bar{\zeta}_{\mathcal{L}}$ lies in $\mathcal{L}_{o p}$. Proposition 7.2. The matrix $\bar{U}$ is the inverse to $U$ in the associative algebra $\operatorname{End}(V) \otimes \tilde{\mathcal{U}}^{*}$. Proof. Let us prove that $\bar{U}$ is a right inverse to $U$. If follows from definition of $*$ and $\bar{U}$ that

$$
U * \bar{U}=\overline{\mathcal{F}}_{1} U \mathcal{F}_{1} \gamma\left(\zeta_{1}\right) \gamma\left(\mathcal{F}_{2}\right) \gamma_{\mathcal{U}^{*}}(U) \gamma \overline{\mathcal{F}}_{2} \gamma(\bar{\zeta}) \otimes \bar{\zeta}_{\mathcal{L}} \overline{\mathcal{F}}_{\mathcal{L}} \otimes \mathcal{F}_{\mathcal{L}} \zeta_{\mathcal{L}}
$$

Note with care that the product of $\mathcal{L}_{o p}$-elements in this formula is written in terms of $\mathcal{L}$. Also mind that we suppressed the symbol of representation $\rho$.

The right-hand side in (72) is equal to unit in view of the identities

$$
\left\{\begin{array}{l}
\mathcal{F}_{1} \gamma\left(\zeta_{1}\right) \gamma\left(\mathcal{F}_{2}\right) \otimes \mathcal{F}_{\mathcal{L}} \zeta_{\mathcal{L}}=1_{\check{\mathcal{U}}} \\
\overline{\mathcal{F}}_{1} \gamma\left(\overline{\mathcal{F}}_{2}\right) \gamma\left(\bar{\zeta}_{1}\right) \otimes \bar{\zeta}_{\mathcal{L}} \overline{\mathcal{F}}_{\mathcal{L}}=1_{\check{\mathcal{U}}}
\end{array}\right.
$$

which hold by definition of $\zeta$, cf. (52).

Now let us prove that $\bar{U}$ is a left inverse to $U$. We have

$$
\bar{U} * U=\gamma(\zeta) \gamma\left(\mathcal{F}_{1}\right) \gamma_{\mathcal{U}^{*}}(U) \gamma\left(\overline{\mathcal{F}}_{1}\right) \gamma(\bar{\zeta}) \Theta_{2^{\prime}} \overline{\mathcal{F}}_{2} U \mathcal{F}_{2} \Theta_{2} \otimes\left(\Theta_{1^{\prime}} \triangleright \bar{\zeta}_{\mathcal{L}}\right) \overline{\mathcal{F}}_{\mathcal{L}} \otimes \mathcal{F}_{\mathcal{L}}\left(\Theta_{1} \triangleright \zeta_{\mathcal{L}}\right) .
$$

Again, we suppressed the symbol or representation $\rho$. The product of $\mathcal{L}_{o p}$-elements is written in terms of $\mathcal{L}$. Remark that $\Theta$-s have appeared in this expression because the first factor $\bar{U}$ contains elements from $\mathcal{L}_{o p} \otimes \mathcal{L}$. Those elements must be pulled through $U$ to the right prior to applying $\mathcal{F}$. This follows from associativity of $*$ and (69).

The right-hand side of the equation (74) is equal to unit if and only if

$$
\left\{\begin{array} { l } 
{ \gamma ( \overline { \mathcal { F } } _ { 1 } ) \gamma ( \overline { \zeta } _ { 1 } ) \Theta _ { 2 } \overline { \mathcal { F } } _ { 2 } \otimes \overline { \mathcal { F } } _ { \mathcal { L } } ( \Theta _ { 1 } \triangleright \overline { \zeta } _ { \mathcal { L } } ) = 1 _ { \check { \mathcal { U } } } } \\
{ \gamma ( \zeta _ { 1 } ) \gamma ( \mathcal { F } _ { 1 } ) \mathcal { F } _ { 2 } \Theta _ { 2 } \otimes \mathcal { F } _ { \mathcal { L } } ( \Theta _ { 1 } \triangleright \zeta _ { \mathcal { L } } ) = 1 _ { \check { \mathcal { U } } } }
\end{array} \Leftrightarrow \left\{\begin{array}{c}
\Gamma(\bar{\zeta})=\vartheta \\
\Gamma(\zeta)=\bar{\vartheta}
\end{array} .\right.\right.
$$

The right system of equations is satisfied by Proposition 6.2. 
Let us associate with the $\mathcal{H}$-invariant matrix $\tilde{S} \in \operatorname{End}^{\otimes 2}(V) \otimes \mathcal{L}$ the extended dFRT algebra $\tilde{\mathcal{T}}_{\text {ext }}$, as in Subsection 3.2. We have the following result, as a corollary of Proposition 7.2 .

Proposition 7.3. The correspondence $T_{j}^{i} \mapsto U_{j}^{i}, \bar{T}_{j}^{i} \mapsto \bar{U}_{j}^{i}$ defines a bialgebroid homomorphism from $\tilde{\mathcal{T}}_{\text {ext }}$ to $\tilde{\mathcal{U}}^{*}$.

Proof. The algebra $\mathcal{U}^{*}$ is a quotient of the FRT algebra $\mathcal{T}$. The matrix $U$ satisfies the relations (71) in $\operatorname{End}(V) \otimes \mathcal{U}^{*}$. Therefore it satisfies the relations (17) in $\operatorname{End}(V) \otimes \tilde{\mathcal{U}}^{*}$, by Proposition 7.1. Further, the matrix $\bar{U}$ is the inverse to $\mathcal{U}$, by Proposition 7.2 These arguments readily imply that the relations (23) involving $\bar{T}$ are also preserved under the correspondence $T \mapsto U, \bar{T} \mapsto \bar{U}$.

\section{3 dRE algebra via dynamical twist}

Denote by $\mathcal{F}_{(12)(34)}$ the image of the dynamical $(\mathcal{U}, \mathcal{H}, \mathcal{L})$-twisting cocycle $\mathcal{F}$ under the map $\Delta \otimes \Delta: \mathcal{U} \otimes \mathcal{U} \rightarrow \mathcal{U}^{\mathcal{R}} \mathcal{U} \otimes \mathcal{U}^{\mathcal{R}} \mathcal{U}$. Since the coproduct $\Delta: \mathcal{U} \rightarrow \mathcal{U}^{\mathcal{R}} \mathcal{U}$ is a Hopf algebra homomorphism, the element $\mathcal{F}_{(12)(34)}$ is a dynamical $\left(\mathcal{U}^{\mathbb{R}} \mathcal{U}, \mathcal{H}, \mathcal{L}\right)$-twisting cocycle. Here $\mathcal{H}$ is viewed as a subalgebra in $\mathcal{U}^{\mathcal{R}} \mathcal{U}$ by the inclusion via $\Delta$. Consider the coboundary

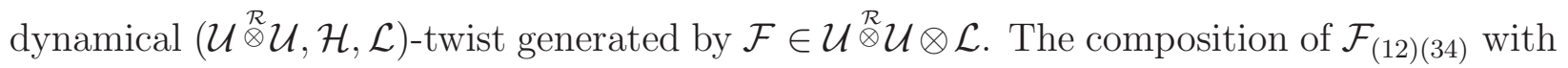
this coboundary twist is again a dynamical $\left(\mathcal{U}^{\mathcal{R}} \mathcal{U}, \mathcal{H}, \mathcal{L}\right)$-twist whose explicit expression is $\left(\mathcal{R}_{23}^{-1} \mathcal{F}_{(13)(24)} \mathcal{R}_{23}\right) \mathcal{F}_{(12)(34)}\left(\mathcal{F}_{12 \mid 34} \mathcal{F}_{34}\right)$. The left factor in the parentheses is obtained by the coproduct of $\mathcal{U}^{\mathcal{R}} \mathcal{U}$ applied to $\mathcal{F}$. The dynamical R-matrix for the triple $(\mathcal{U} \otimes \mathcal{R}, \mathcal{H}, \mathcal{L})$ then reads

$$
\left(\mathcal{F}_{(34)(12)} \mathcal{F}_{34 \mid 12} \mathcal{F}_{12}\right)^{-1}\left(\mathcal{R}_{14}^{-} \mathcal{R}_{24} \mathcal{R}_{13}^{-} \mathcal{R}_{23}\right)\left(\mathcal{F}_{(12)(34)} \mathcal{F}_{12 \mid 34} \mathcal{F}_{34}\right)=\tilde{\mathcal{R}}_{14 \mid 2}^{-} \tilde{\mathcal{R}}_{24} \tilde{\mathcal{R}}_{13 \mid 24}^{-} \tilde{\mathcal{R}}_{23 \mid 4}
$$

where $\tilde{\mathcal{R}}=\overline{\mathcal{F}}_{21} \mathcal{R} \mathcal{F}$ and $\tilde{\mathcal{R}}^{-}=\overline{\mathcal{F}}_{21} \mathcal{R}^{-} \mathcal{F}=\tilde{\mathcal{R}}_{21}^{-1}$. Let us perform one more coboundary twist with the element $\zeta_{1 \mid 2} \in \mathcal{U}^{\mathcal{R}} \mathcal{U} \otimes \mathcal{L}$. Denote by $\Upsilon$ the resulting composite dynamical twist.

Let $\mathcal{K}$ be the RE algebra associated with $S$ and generated by the matrix elements $\left\|K_{j}^{i}\right\|$. It is a module algebra over $\mathcal{U}^{\mathcal{R}} \mathcal{U}$ with respect to the action

$$
(x \otimes y) \triangleright K=\rho(\gamma(x)) K \rho(y), \quad x \otimes y \in \mathcal{U}^{\mathcal{R}} \mathcal{U} .
$$

Since $\mathcal{U}^{\mathcal{R}} \mathcal{U}$ coincides as an algebra with $\mathcal{U} \otimes \mathcal{U}$, this action can be expressed through right and left actions of $\mathcal{U}$

$$
(x \otimes y) \triangleright A=y \rightarrow A<\gamma(x) .
$$


Remark that $\mathcal{K}$ coincides as a linear space with the FRT algebra $\mathcal{T}$, which is a bialgebra and admits a Hopf pairing with $\mathcal{U}$. The actions $\rightarrow$ and $<$ turns into, respectively, left and right coregular actions of $\mathcal{U}$ on $\mathcal{T}$.

Consider $\mathcal{K} \otimes \mathcal{L}$ as an algebra over the left $\mathcal{L}$-bialgebroid $\left(\mathcal{U}^{\mathcal{R}} \mathcal{U}\right) \otimes\left(\mathfrak{D H} \mathcal{H}_{\mathcal{L}}\right)$. The bialgebroid twist induced by $\Upsilon$ transforms $\mathcal{K} \otimes \mathcal{L}$ to a module algebra, $\tilde{\mathcal{K}}^{\prime \prime}$, over the twisted bialgebroid. Let $\tilde{S}$ be the dynamical matrix built by $\tilde{\mathcal{R}}$ as in Subsection 7.2 Associate with $\tilde{S}$ the dRE algebra $\tilde{\mathcal{K}}$, according to Definition 3.9

Theorem 7.4. The algebra $\tilde{\mathcal{K}}^{\prime \prime}$ is isomorphic to the dRE algebra $\tilde{\mathcal{K}}$.

Proof. The dynamical twist $\Upsilon$ converts the universal R-matrix of $\mathcal{U}^{\mathcal{R}} \mathcal{U}$ to the dynamical R-matrix

$$
\left(\bar{\zeta}_{3 \mid 412} \bar{\zeta}_{1 \mid 2}\right) \tilde{\mathcal{R}}_{14 \mid 2}^{-} \tilde{\mathcal{R}}_{24} \tilde{\mathcal{R}}_{13 \mid 24}^{-} \tilde{\mathcal{R}}_{23 \mid 4}\left(\zeta_{1 \mid 234} \zeta_{3 \mid 4}\right) \in\left(\mathcal{U}^{\mathcal{R}} \mathcal{U}\right) \otimes\left(\mathcal{U}^{\mathcal{R}} \mathcal{U}\right) \otimes \mathcal{L}
$$

To write out the commutation relations in $\tilde{\mathcal{K}}^{\prime \prime}$, it is convenient to represent this $\mathrm{R}$-matrix in the form (recall that the bar means the inverse)

$$
\mathcal{S}_{21}^{\prime} \overline{\mathcal{S}}^{\prime \prime}=\left(\bar{\zeta}_{3 \mid 412} \bar{\zeta}_{1 \mid 2}\right) \tilde{\mathcal{R}}_{14 \mid 2}^{-} \tilde{\mathcal{R}}_{24}\left(\zeta_{3 \mid 24} \zeta_{1 \mid 324}\right)\left(\bar{\zeta}_{1 \mid 324} \bar{\zeta}_{3 \mid 24}\right) \tilde{\mathcal{R}}_{13 \mid 24}^{-} \tilde{\mathcal{R}}_{23 \mid 4}\left(\zeta_{3 \mid 4} \zeta_{1 \mid 234}\right) .
$$

where $\mathcal{S}^{\prime}=\mathcal{S}_{1}^{\prime} \otimes \mathcal{S}_{2}^{\prime} \otimes \mathcal{S}_{\mathcal{L}}^{\prime}$ and $\mathcal{S}^{\prime \prime}=\mathcal{S}_{1}^{\prime \prime} \otimes \mathcal{S}_{2}^{\prime \prime} \otimes \mathcal{S}_{\mathcal{L}}^{\prime \prime}$ belong to $\left(\mathcal{U}^{\mathcal{R}} \mathcal{U}\right) \otimes\left(\mathcal{U}^{\mathcal{R}} \mathcal{U}\right) \otimes \mathcal{L}$ and

$$
\begin{aligned}
& \mathcal{S}^{\prime}:=\left(\bar{\zeta}_{1 \mid 234} \bar{\zeta}_{3 \mid 4}\right) \tilde{\mathcal{R}}_{23 \mid 4}^{-1} \tilde{\mathcal{R}}_{42}\left(\zeta_{1 \mid 42} \zeta_{3 \mid 142}\right)=\left(\bar{\zeta}_{3 \mid 4} \tilde{\mathcal{R}}_{23 \mid 4}^{-1} \zeta_{3 \mid 24}\right)\left(\tilde{\mathcal{R}}_{42}\right) \\
& \mathcal{S}^{\prime \prime}:=\left(\bar{\zeta}_{1 \mid 234} \bar{\zeta}_{3 \mid 4}\right) \tilde{\mathcal{R}}_{23 \mid 4}^{-1} \tilde{\mathcal{R}}_{31 \mid 24}\left(\zeta_{3 \mid 42} \zeta_{1 \mid 324}\right)=\left(\bar{\zeta}_{3 \mid 4} \tilde{\mathcal{R}}_{23 \mid 4}^{-1} \zeta_{3 \mid 24}\right)\left(\bar{\zeta}_{3 \mid 24} \bar{\zeta}_{1 \mid 324} \tilde{\mathcal{R}}_{31 \mid 24} \zeta_{1 \mid 24} \zeta_{3 \mid 124}\right)
\end{aligned}
$$

Then the commutation relation (170) for two arbitrary elements $A, B \in \mathcal{K} \subset \tilde{\mathcal{K}}^{\prime \prime}$ can be written as

$$
\left(\mathcal{S}_{1}^{\prime} \triangleright B\right) *\left(\mathcal{S}_{2}^{\prime} \triangleright A\right) * \mathcal{S}_{\mathcal{L}}^{\prime}=\left(\mathcal{S}_{1}^{\prime \prime} \triangleright A\right) *\left(\mathcal{S}_{2}^{\prime \prime} \triangleright B\right) * \mathcal{S}_{\mathcal{L}}^{\prime \prime},
$$

The left equalities (61), (62), and (63) can be represented as

$$
\begin{aligned}
(\gamma \otimes \gamma)\left(\bar{\zeta}_{2 \mid 1} \bar{\zeta}_{1} \tilde{\mathcal{R}} \zeta_{1 \mid 2} \zeta_{2}\right) & =\left(\bar{\gamma}\left(\Theta_{1}\right) \triangleright \tilde{\mathcal{R}}\right) \Delta\left(\Theta_{2}\right) \\
(\gamma \otimes \mathrm{id})\left(\bar{\zeta}_{1} \tilde{\mathcal{R}} \zeta_{1 \mid 2}\right) & =\left(\bar{\gamma}\left(\Theta_{1}\right) \triangleright \tilde{\mathcal{R}}^{-1}\right)\left(\Theta_{2} \otimes 1\right) \\
(\mathrm{id} \otimes \gamma)\left(\bar{\zeta}_{2} \tilde{\mathcal{R}}^{-1} \zeta_{2 \mid 1}\right) & =\left(\bar{\gamma}\left(\Theta_{1}\right) \triangleright \tilde{\mathcal{R}}\right)\left(1 \otimes \Theta_{2}\right)
\end{aligned}
$$

We substitute (77) into (78) and evaluate the action (76)). The result will be expressed trough the left hand-side of (179)-(81). Then we replace them by the right-hand side of (179)-(81). In that way we have for the right-hand side of (78)

$$
\left\{\tilde{\mathcal{R}}_{1^{\prime}} \Theta_{2}^{(3)} \rightarrow A<\tilde{\mathcal{R}}_{2} \gamma\left(\Theta_{2}^{(1)}\right)\right\} * \tilde{\mathcal{R}}_{\mathcal{L}^{\prime}} *\left\{\Theta_{2}^{(4)} \rightarrow B<\tilde{\mathcal{R}}_{1} \gamma\left(\Theta_{2}^{(2)}\right) \tilde{\mathcal{R}}_{2^{\prime}}\right\} *\left\{\Theta_{1} \triangleright \tilde{\mathcal{R}}_{\mathcal{L}}\right\} .
$$


The dynamical R-matrix has the symmetry type $(\tau, \epsilon)$. Applying this argument to the R-matrix labelled with prime, we rewrite (82) as

$$
\begin{aligned}
\left\{\Theta_{2}^{(2)} \tilde{\mathcal{R}}_{1^{\prime}} \rightarrow A<\tilde{\mathcal{R}}_{2} \gamma\left(\Theta_{2}^{(1)}\right)\right\} & *\left\{\Theta_{2}^{(3)} \triangleright \tilde{\mathcal{R}}_{\mathcal{L}^{\prime}}\right\} * \\
& *\left\{\Theta_{2}^{(5)} \rightarrow B \leftarrow \tilde{\mathcal{R}}_{1} \tilde{\mathcal{R}}_{2^{\prime}} \gamma\left(\Theta_{2}^{(4)}\right)\right\} *\left\{\Theta_{1} \triangleright \tilde{\mathcal{R}}_{\mathcal{L}}\right\}
\end{aligned}
$$

Now recall that elements of $\mathcal{K}$ and $\mathcal{L}$ permute in the algebra $\tilde{\mathcal{K}}$ according to the rule

$$
\lambda * A=\left(\Theta_{2}^{(2)} \rightarrow A<\gamma\left(\Theta_{2}^{(1)}\right)\right) *\left(\Theta_{1} \triangleright \lambda\right)
$$

for all $A \in \mathcal{K}$ and $\lambda \in \mathcal{L}$ (apply (69) and (76) setting $x \otimes y=\Delta\left(\Theta_{2}\right)$ ). Taking this into account, pull the factor $\Theta_{1} \triangleright \tilde{\mathcal{R}}_{\mathcal{L}}$ in (83) to the left. This will kill all the $\Theta$-s. Thus the right-hand side of (78) acquires the form

$$
\tilde{\mathcal{R}}_{\mathcal{L}} *\left(\tilde{\mathcal{R}}_{1^{\prime}} \rightarrow A<\tilde{\mathcal{R}}_{2}\right) * \tilde{\mathcal{R}}_{\mathcal{L}^{\prime}} *\left(B<\tilde{\mathcal{R}}_{1} \tilde{\mathcal{R}}_{2^{\prime}}\right)
$$

Taking $A$ and $B$ to be the matrix coefficients $\left\|K_{j}^{i}\right\|$, we obtain the left-hand side of (32).

For the left-hand side of (178) we have

$$
\begin{gathered}
\left\{\tilde{\mathcal{R}}_{1} \tilde{\mathcal{R}}_{2^{\prime}} \rightarrow B\right\} *\left\{\Theta_{2}^{(2)} \tilde{\mathcal{R}}_{1^{\prime}} \rightarrow A<\tilde{\mathcal{R}}_{2} \gamma\left(\Theta_{2}^{(1)}\right)\right\} *\left\{\Theta_{1} \triangleright \tilde{\mathcal{R}}_{\mathcal{L}}\right\} * \tilde{\mathcal{R}}_{\mathcal{L}^{\prime}}= \\
=\left\{\tilde{\mathcal{R}}_{1} \tilde{\mathcal{R}}_{2^{\prime}} \rightarrow B\right\} *\left\{\tilde{\mathcal{R}}_{\mathcal{L}}\right\} *\left\{\tilde{\mathcal{R}}_{1^{\prime}} \rightarrow A \leftarrow \tilde{\mathcal{R}}_{2}\right\} * \tilde{\mathcal{R}}_{\mathcal{L}^{\prime}} .
\end{gathered}
$$

Taking $A$ and $B$ to be the matrix coefficients $\left\|K_{j}^{i}\right\|$ we obtain the right-hand side of (32).

Thus we have shown that there is an algebra map $\tilde{\mathcal{K}} \rightarrow \tilde{\mathcal{K}}^{\prime \prime}$. This map is in fact an isomorphism. The ordinary RE algebra is the quotient of $k\left\langle K_{j}^{i}\right\rangle$ by the RE relations. The bialgebroid twist $\Upsilon$ transforms the algebra $k\left\langle K_{j}^{i}\right\rangle \otimes \mathcal{L}$, which is free over $\mathcal{L}$, into an $\mathcal{L}$-free algebra. It is easy to see that that algebra is isomorphic to $\mathfrak{F}(K)=k\left\langle K_{j}^{i}\right\rangle \rtimes \mathcal{L}$. Therefore $\tilde{\mathcal{K}}^{\prime \prime}$ is a quotient of $\mathfrak{F}(K)$ by the dRE relations, which are the $\Upsilon$-image of the ordinary RE relations. Hence $\tilde{\mathcal{K}}^{\prime \prime} \simeq \tilde{\mathcal{K}}$.

\subsection{A homomorphism of the dRE algebra to $\mathcal{U} \otimes \mathcal{L}$}

The ordinary RE algebra associated with a representation of a quasitriangular Hopf algebra $\mathcal{U}$ admits a homomorphism to $\mathcal{U}$. Below we show that the dRE algebra has a homomorphism into $\check{\mathcal{U}}$.

First of all observe that the matrix $\tilde{\mathcal{Q}}:=\mathcal{R}_{21} \mathcal{R} \in{ }_{\epsilon} \check{\mathcal{U}}_{\epsilon}^{2}$ satisfies equation

$$
\tilde{\mathcal{R}}_{21 \mid 3} \tilde{\mathcal{Q}}_{13} \tilde{\mathcal{R}}_{12 \mid 3} \tilde{\mathcal{Q}}_{23}=\tilde{\mathcal{Q}}_{23} \tilde{\mathcal{R}}_{21 \mid 3} \tilde{\mathcal{Q}}_{13} \tilde{\mathcal{R}}_{12 \mid 3}
$$


in $\check{\mathcal{U}}^{3}$. This equation can be derived directly from the dYBE. Another way is to interpret the dynamical R-matrix through the generator of a non-local representation of the braid group. Put $\tilde{Q}=\rho\left(\tilde{\mathcal{Q}}_{1}\right) \otimes \tilde{\mathcal{Q}}_{2} \otimes \tilde{\mathcal{Q}}_{\mathcal{L}} \in \operatorname{End}(V) \otimes \mathcal{U} \otimes \mathcal{L}=\operatorname{End}(V) \otimes \check{\mathcal{U}}$.

Proposition 7.5. Along with the embedding $\delta: \mathcal{L} \rightarrow \mathcal{U} \otimes \mathcal{L}$, the correspondence $K_{j}^{i} \mapsto \tilde{Q}_{j}^{i}$ defines an algebra homomorphism $\varphi: \tilde{\mathcal{K}} \rightarrow \check{\mathcal{U}}$.

Proof. Applying $\rho \otimes \rho$ to the first two $\mathcal{U}$-factors in (84), we come to the equation

$$
\tilde{R}_{21 \mid 3} \tilde{Q}_{13} \tilde{R}_{12 \mid 3} \tilde{Q}_{23}=\tilde{Q}_{23} \tilde{R}_{21 \mid 3} \tilde{Q}_{13} \tilde{R}_{12 \mid 3} .
$$

This is exactly the image of the dRE equation (32) under the map $\varphi$. We must to check that the map $\varphi$ preserves the permutation rule (31). This is the case due to $\tilde{\mathcal{Q}} \in{ }_{\epsilon} \check{\mathcal{U}}_{\epsilon}^{2}$.

Remark 7.6. Equation (85) is equivalent to the dRE considered in [FHS for the case of abelian base. Indeed, multiply equation (844) by $\zeta_{1 \mid 23} \zeta_{2 \mid 3} \zeta_{3}$ from the right and by $\bar{\zeta}_{1 \mid 23} \bar{\zeta}_{2 \mid 3} \bar{\zeta}_{3}$ from the left, then apply $\Gamma_{123}$. One can check, using Proposition 6.4 (compare this also with Example 5.5), that this operation converts (84) into

$$
\tilde{\mathcal{Q}}_{23 \mid 1} \tilde{\mathcal{R}}_{21} \tilde{\mathcal{Q}}_{13 \mid 2} \tilde{\mathcal{R}}_{12}=\tilde{\mathcal{R}}_{21} \tilde{\mathcal{Q}}_{13 \mid 2} \tilde{\mathcal{R}}_{12} \tilde{\mathcal{Q}}_{23 \mid 1}
$$

Passing to a representation we come to the dRE that appeared in [FHS].

\section{Dynamical trace and center of the dRE algebra}

\subsection{Dynamical trace}

In this section we introduce dynamical trace (in the twisted setting) and use it to construct central elements of the dRE algebra.

Set $\mu:=\mathcal{R}_{1} \gamma\left(\mathcal{R}_{2}\right) \in \mathcal{U}$. The conjugation with $\mu$ implements the inverse squared antipode in $\mathcal{U}$, Dr2]. Let $(V, \rho)$ be an $\mathcal{U}$-module and let $\mathcal{A}$ be a module over the left bialgebroid $\mathcal{L} \rtimes \mathcal{H}$. Then $\mathcal{A}$ is an $\mathcal{L}$-bimodule. The right $\mathcal{L}$-action is expressed through the left $\mathcal{L}$-action and the action $\bullet$ of $\mathcal{H}$ by $\lambda a=\left(\lambda^{(1)}-a\right) \lambda^{[\infty]}$, where $a \in \mathcal{A}$ and $\lambda \in \mathcal{L}$. We will say that a matrix $X \in \operatorname{End}(V) \otimes \mathcal{A}$ is $\mathcal{H}$-equivariant if its coefficients form the dual module to $\operatorname{End}(V)$, namely $h \triangleright X=\rho\left(\gamma\left(h^{(1)}\right)\right) X \rho\left(h^{(2)}\right)$, for all $h \in \mathcal{H}$.

To simplify further formulas we will suppress the symbol of representation $\rho$ whenever possible. 
Definition 8.1. Let $X$ an $\mathcal{H}$-invariant matrix with coefficients in $\mathcal{A}$. Dynamical trace $\operatorname{Tr}_{d}(X)$ is the element of $\mathcal{A}$ defined by

$$
\operatorname{Tr}_{d}(X):=\operatorname{Tr}(\mu \vartheta X \zeta)
$$

Formula (87) gives a generalization to the dynamical case of the quantum trace for a quasitriangular Hopf algebra and its twist. Let us prove the following important properties of this generalization.

Lemma 8.2. The dynamical trace satisfies the following equalities:

$$
\operatorname{Tr}(X \zeta \bar{\Gamma}(\bar{\zeta}) \mu)=\operatorname{Tr}_{d}(X)=\operatorname{Tr}(\mu \Gamma(\bar{\vartheta}) \vartheta X)
$$

Proof. We will prove only the right equality in (88); the left one is verified similarly. If the coefficients of the matrix $\rho(\zeta)$ were scalars, we could have moved $\zeta$ in $\operatorname{Tr}(\mu \vartheta X \zeta)$ freely to the left-most position. However we should take into account how elements from $\mathcal{L}$ permute with the coefficients of the matrix $\rho(\vartheta) X$. The latter is $\mathcal{H}$-invariant, and we can transform $\operatorname{Tr}(\mu \vartheta X \zeta)$ to

$$
\left.(\operatorname{Tr} \otimes \mathrm{id})\left(\bar{\gamma}\left(\Theta_{2^{\prime}}\right) \zeta_{1} \mu \vartheta_{1} \Theta_{2} \otimes \vartheta_{\mathcal{L}}\left(\left(\Theta_{1} \Theta_{1^{\prime}}\right) \triangleright \zeta_{\mathcal{L}}\right)\right) \sum_{i, j} e_{j}^{i} \otimes X_{i}^{j}\right) .
$$

As an element from ${ }_{\epsilon} \check{\mathcal{U}}_{\epsilon}, \vartheta$ commutes with $\delta(\mathcal{L})$. Therefore the factor before the sum is equal to $\left.\bar{\gamma}\left(\Theta_{2^{\prime}}\right) \zeta_{1} \mu \Theta_{2} \vartheta_{1} \otimes\left(\left(\Theta_{1} \Theta_{1^{\prime}}\right) \triangleright \zeta_{\mathcal{L}}\right)\right) \vartheta_{\mathcal{L}} \in \check{\mathcal{U}}$. Pulling $\mu$ to the left we bring this expression to $\mu \Gamma^{2}(\zeta) \vartheta=\mu \Gamma(\bar{\vartheta}) \vartheta$. This proves the right equality in (88).

Lemma 8.3. Let $X \in \operatorname{End}(V) \otimes \mathcal{A}$ be an $\mathcal{H}$-invariant matrix. Then

$$
\operatorname{Tr}_{1}\left(\mu_{1} \vartheta_{1 \mid 2} \tilde{\mathcal{R}}_{12}^{-1} X_{1} \tilde{\mathcal{R}}_{12} \zeta_{1 \mid 2}\right)=1_{\operatorname{End}(V)} \otimes \operatorname{Tr}_{d}(X)=\operatorname{Tr}_{1}\left(\mu_{1} \vartheta_{1 \mid 2} \tilde{\mathcal{R}}_{21} X_{1} \tilde{\mathcal{R}}_{21}^{-1} \zeta_{1 \mid 2}\right)
$$

Proof. Using (62) and (63) we rewrite (90) as

$$
\operatorname{Tr}_{1}\left(\mu_{1} \Gamma_{1}\left(\tilde{\mathcal{R}}_{12}\right) \vartheta_{1} X_{1} \zeta_{1} \bar{\Gamma}_{1}\left(\tilde{\mathcal{R}}_{12}^{-1}\right)\right)=1 \otimes \operatorname{Tr}_{d}(X)=\operatorname{Tr}_{1}\left(\mu_{1} \Gamma_{1}\left(\tilde{\mathcal{R}}_{21}^{-1}\right) \vartheta_{1} X_{1} \zeta_{1} \bar{\Gamma}\left(\tilde{\mathcal{R}}_{21}\right)\right) .
$$

We will check only the left equality in (91). We will see that it holds for an arbitrary matrix $\tilde{R}$, therefore our proof will be valid upon replacement $\tilde{R} \leftrightarrow \tilde{R}_{21}^{-1}$.

Pull the matrix $\bar{\Gamma}_{1}\left(\tilde{\mathcal{R}}_{12}^{-1}\right)$ to the left under the trace taking into account how elements from $\mathcal{L}$ permutes with coefficients of the matrix $\rho(\vartheta) X \rho(\zeta)$ (cf. the proof of Proposition 8.2). The latter is $\mathcal{H}$-invariant, therefore the expression under $\operatorname{Tr}_{1}$ can be brought to the form

$$
\left(\bar{\gamma}\left(\Theta_{2^{\prime \prime}}\right) \Theta_{2^{\prime}} \bar{\gamma}\left(\tilde{\mathcal{R}}_{1^{\prime}}^{-1}\right) \mu \gamma\left(\bar{\Theta}_{2} \tilde{\mathcal{R}}_{1}\right) \Theta_{2^{\prime \prime \prime}} \otimes \tilde{\mathcal{R}}_{2} \tilde{\mathcal{R}}_{2^{\prime}}^{-1} \otimes\left(\bar{\Theta}_{1} \triangleright \mathcal{R}_{\mathcal{L}}\right)\left(\left(\Theta_{1^{\prime \prime \prime}} \Theta_{1^{\prime \prime}} \Theta_{1^{\prime}}\right) \triangleright \mathcal{R}_{\mathcal{L}^{\prime}}^{-1}\right)\right)\left(\vartheta_{1} X_{1} \zeta_{1}\right)
$$


(mind that we suppress $\rho$ ). We will prove the lemma if show that the left factor within the big parentheses equals $\mu_{1} \in \check{\mathcal{U}}^{2}$. First of all notice that the term $\bar{\gamma}\left(\Theta_{2^{\prime \prime}}\right) \Theta_{2^{\prime}} \otimes \Theta_{1^{\prime \prime}} \Theta_{1^{\prime}}$ cancels. Then pull $\mu$ to the right and employ the fact that conjugation with $\mu$ implements $\gamma^{-2}$. The resulting expression in the big brackets will be

$$
\bar{\gamma}\left(\tilde{\mathcal{R}}_{1^{\prime}}^{-1}\right) \bar{\gamma}\left(\bar{\Theta}_{2} \tilde{\mathcal{R}}_{1}\right) \bar{\gamma}\left(\bar{\Theta}_{2^{\prime \prime \prime}}\right) \mu \otimes \tilde{\mathcal{R}}_{2} \tilde{\mathcal{R}}_{2^{\prime}}^{-1} \otimes\left(\bar{\Theta}_{1} \triangleright \tilde{\mathcal{R}}_{\mathcal{L}}\right)\left(\bar{\Theta}_{1^{\prime \prime \prime}} \triangleright \tilde{\mathcal{R}}_{\mathcal{L}^{\prime}}^{-1}\right),
$$

which is equal to

$$
\bar{\gamma}\left(\tilde{\mathcal{R}}_{1} \tilde{\mathcal{R}}_{1^{\prime}}^{-1}\right) \bar{\gamma}\left(\bar{\Theta}_{2}\right) \mu \otimes \tilde{\mathcal{R}}_{2} \tilde{\mathcal{R}}_{2^{\prime}}^{-1} \otimes \bar{\Theta}_{1} \triangleright\left(\tilde{\mathcal{R}}_{\mathcal{L}} \tilde{\mathcal{R}}_{\mathcal{L}^{\prime}}^{-1}\right)=\mu_{1}
$$

Thus the leftmost term in (90) equals $\operatorname{Tr}_{1}\left(\mu_{1} \vartheta_{1} X_{1} \zeta_{1}\right)$, which proves the left quality.

Remark 8.4. The dynamical trace can be defined for any (dualizable) solution to dYBE, without the dynamical twist assumption. Indeed, such solution (multiplied by permutation) is just a Yang-Baxter operator in the dynamical category, which is rigid, cf. Remark 6.5 For the theory of dualizable Yang-Baxter operators in rigid categories, consult e.g. [JS].

\subsection{On the center of the dRE algebra}

We will apply the dynamical trace to constructing central elements of the algebra $\tilde{\mathcal{K}}$. We set $\mathcal{A}=\tilde{\mathcal{K}}$ and notice that any power $K^{n}, n \in \mathbb{N}$, of the matrix $K$ is $\mathcal{H}$-invariant.

Theorem 8.5. Let $K$ be the matrix of the generators of the dRE algebra $\tilde{\mathcal{K}}$. Then for all $n \in \mathbb{N}$ the elements $\operatorname{Tr}_{d}\left(K^{n}\right)$ belong to the center of $\tilde{\mathcal{K}}$.

Proof. The dRE implies the following equation for any matrix power $K^{n}$ :

$$
\tilde{R}_{12}^{-1} K_{1}^{n} \tilde{R}_{12} K_{2}=K_{2} \tilde{R}_{21} K_{1}^{n} \tilde{R}_{21}^{-1}
$$

Multiply this equation by $\mu_{1} \vartheta_{1 \mid 2}$ from the left and by $\zeta_{1 \mid 2}$ from the right. Since $K$ is $\mathcal{H}$ invariant, the matrices $\mathcal{K}_{2}$ and $\xi_{1 \mid 2}$ commute for any $\xi \in \operatorname{End}(V) \otimes \mathcal{L}$. Now apply Lemma 8.3

Let us look at how the center $\mathcal{K}$ of the $\mathrm{RE}$ algebra $\mathcal{K}$ transforms under the dynamical twist. It is known that all $\mathcal{U}$-invariant elements of $\mathcal{K}$ lie in the center. Let us say a few words about the nature of this phenomenon. The $\mathcal{U}$-module structure on $\mathcal{K}$ is induced by the Hopf algebra embedding $\mathcal{U} \stackrel{\Delta}{\longrightarrow} \mathcal{U}^{\mathcal{R}} \mathcal{U}$. The algebra $\mathcal{K}$ is commutative in the category of $\mathcal{U}^{\mathcal{R}} \mathcal{U}$-modules. One tensor factor of the universal R-matrix of $\mathcal{U}^{\mathcal{R}} \mathcal{U}$ lies in the image 
$\Delta(\mathcal{U}) \subset \mathcal{U}^{\mathcal{R}} \mathcal{U}$. Therefore the universal R-matrix reduces to unit whenever that factor acts on a $\mathcal{U}$-invariant. Thus any such element commutes with entire $\mathcal{K}$.

Similar effect takes place when a quasitriangular Hopf algebra is replaced by a quasitriangular bialgebroid. Let us specialize our consideration to the dRE algebra. Identify $\mathcal{K}$ with a subspace $\mathcal{K} \otimes 1 \subset \tilde{\mathcal{K}}$ and consider $\tilde{\mathcal{K}}$ as a natural module $\simeq \mathcal{K} \otimes \mathcal{L}$ over the associative algebra $(\mathcal{U} \otimes \mathcal{U}) \otimes \mathcal{L}$.

Proposition 8.6. For any $\mathcal{U}$-invariant element $a \in \mathcal{K}$ the element

$$
\left((\zeta \bar{\Gamma}(\bar{\zeta}))_{1} \rightarrow a\right) \otimes(\zeta \bar{\Gamma}(\bar{\zeta}))_{\mathcal{L}}
$$

is central in $\tilde{\mathcal{K}}$.

Proof. Consider the algebra $\tilde{\mathcal{K}}^{\prime}$ obtained from $\mathcal{K}$ by the dynamical twist $\mathcal{F}_{(12)(34)}$, as in Subsection [7.3. Multiplication in $\tilde{\mathcal{K}}^{\prime}$ (as well as in $\tilde{\mathcal{K}}$ ) makes it a free right $\mathcal{L}$-module $\simeq \mathcal{K} \mathcal{L}$. Therefore, to prove centrality of an element, it suffices to show that it commutes with elements from $\mathcal{K}$ and $\mathcal{L}$.

Every $\mathcal{U}$-invariant element $a$ from $\mathcal{K}$ is $\mathcal{H}$-invariant and therefore commutes with $\mathcal{L}$ in the algebra $\tilde{\mathcal{K}}^{\prime}$, cf. (69). Let us show that it also commutes with elements from $\mathcal{K} \subset \tilde{\mathcal{K}}^{\prime}$. Indeed, the permutation of $a$ with elements from $\mathcal{K}$ is controlled by the dynamical $\mathrm{R}$-matrix, cf. (170). In our case that dynamical R-matrix is obtained from the R-matrix of $\mathcal{U} \stackrel{\mathcal{R}}{\mathcal{U}}$ by dynamical twist $\mathcal{F}_{(13)(24)}$. Both $\mathcal{R}$ and $\mathcal{F}_{(13)(24)}$ cancel on $a$. Therefore a commutes with $\mathcal{K}$ and is thus central in $\tilde{\mathcal{K}}^{\prime}$.

By Theorem 7.4, the algebras $\tilde{\mathcal{K}}$ and $\tilde{\mathcal{K}}^{\prime}$ are module algebras over bialgebroids that differ from each other by the coboundary twist $\mathcal{F}_{12} \zeta_{1 \mid 2} \in \mathcal{U}^{\mathcal{R}} \mathcal{U} \otimes \mathcal{L} \subset \mathcal{U}^{\mathcal{R}} \mathcal{U} \otimes \mathfrak{D H} \mathcal{H}_{\mathcal{L}}$. Therefore $\tilde{\mathcal{K}}^{\prime}$ and $\tilde{\mathcal{K}}$ are isomorphic as associative algebras, and that isomorphism is implemented via the element $\mathcal{F}_{12} \zeta_{1 \mid 2}$ :

$$
\tilde{\mathcal{K}}^{\prime} \ni b \mapsto\left(\mathcal{F}_{12} \zeta_{1 \mid 2}\right)^{-1} \triangleright b \in \tilde{\mathcal{K}}
$$

Here we denote the action of $\mathcal{U}^{\mathcal{R}} \mathcal{U} \otimes \mathfrak{D} \mathcal{H}_{\mathcal{L}}$ by the same symbol as the action of $\mathcal{U} \otimes \mathcal{R}$. To finish the proof, one should evaluate this map taking into account

$$
(x \otimes y) \triangleright a=(y \gamma(x)) \rightarrow a
$$

for any $\mathcal{U}$-invariant element $a \in \mathcal{K} \subset \tilde{\mathcal{K}}$ and any $x \otimes y \in \mathcal{U}^{\mathcal{R}} \mathcal{U}$.

Remark 8.7. Proposition 8.6 tells us that the center of $\tilde{\mathcal{K}}$ is not less than the center of $\mathcal{K}$. However, Proposition 8.6 does not directly imply Theorem 8.5 
Acknowledgement. A. Mudrov is grateful to the Max-Planck Institut für Mathematik for hospitality. He appreciates numerous useful and interesting discussions with P. Pyatov. He also thanks D. Gurevich for drawing attention to the problem of dynamical trace and center of the dynamical reflection equation algebra.

\section{References}

[ABB] J. Avan, O. Babelon, and E. Billey: The Gervais-Neveu-Felder equation and the quantum Calogero-Moser systems, Commun. Math. Phys. 178 (1996) 281 - 299.

[AF] A. Alekseev and L. Faddeev: $T^{*}(G)_{t}$ : a toy model of conformal field theory, Commun. Math. Phys. 141 (1991) 413-422.

[Dr1] V. Drinfeld: Quantum Groups, in Proc. Int. Congress of Mathematicians, Berkeley, 1986, ed. A. V. Gleason, AMS, Providence (1987) 798-820.

[Dr2] V. Drinfeld: Almost cocommutative Hopf algebras, Leningrad Math. J. 2 (1990), \# 2, $321-342$.

[Dr3] V. Drinfeld: Quasi-Hopf algebras, Leningrad Math. J. 1 (1990), \# 6, 1419-1457.

[DM1] J. Donin and A. Mudrov: Dynamical Yang-Baxter equation and quantum vector bundles, arXiv: math.QA/0306028.

[DM2] J. Donin and A. Mudrov: Quantum groupoids associated with dynamical categories, math.QA/0311316.

[DM3] J. Donin and A. Mudrov: Reflection Equation, Twist, and Equivariant Quantization, Isr. J. Math., 136 (2003) 11-28.

[EE] B. Enriquez and P. Etingof: Quantization of classical dynamical r-matrices with nonabelian base, arXiv:math.QA/0311224.

[EEM] B. Enriquez, P. Etingof, and I. Marshall: Quantization of some Poisson-Lie dynamical r-matrices and Poisson homogeneous spaces, arXiv:math.QA/0403283.

[ES2] P. Etingof and O. Schiffmann: On the moduli space of classical dynamical r-matrices, Math. Res. Lett. 9 (2001) 157-170. 
[ES1] P. Etingof and O. Schiffmann: Lectures on the dynamical Yang-Baxter equation. Quantum Groups and Lie theory, London Math. Soc. Lecture Note 290 (2001) 89-129.

[ESS] P. Etingof, O. Schiffmann, and T. Schedler: Explicit quantization of dynamical rmatrices for finite dimensional semisimple Lie algebras, J.AMS 13 (2000) 595-609.

[EV1] P. Etingof and A. Varchenko: Solutions of the quantum dynamical Yang-Baxter equation and dynamical quantum groups, Commun. Math. Phys. 196 (1998) 591-640.

[EV2] P. Etingof and A. Varchenko: Exchange dynamical quantum groups, Commun. Math. Phys. 205 (1999) 19-52.

[F] G. Felder: Conformal field theories and integrable models associated to elliptic curves, Proc. ICM Zurich, (1994) 1247-1255.

[Fad] L. Faddeev: On the exchande matrix of the WZNW model, Commun. Math. Phys. $132(1990)$ 131-138.

[FHS] H. Fan, B.-Y. Hou, and K. Shi: Representation of the boundary elliptic quantum group $\mathcal{B} E_{\tau, \eta}\left(s l_{2}\right)$ and the Bethe ansatz, Nucl. Phys. B 496 (1997) 551-570.

[FRT] L. Faddeev, N. Reshetikhin, and L. Takhtajan: Quantization of Lie groups and Lie algebras, Leningrad Math. J. 1 (1990) 193-226.

[GN] J.-L. Gervais and A. Neveu: Novel triangle relation and absence of tachyons in Liouville string theory, Nucl. Phys. B 238 (1984) 125-141.

[JS] A. Joyal and R. Street An introduction to Tannaka duality and quantum groups. Lecture Notes in Math. 1488 (1991) 413-492.,

[KS] P. P. Kulish and R. Sasaki: Covariance properties of reflection equation algebras, Prog. Theor. Phys. 89 \#3 (1993) 741-761.

[KSkl] P. P. Kulish and E. K. Sklyanin: Algebraic structure related to the reflection equation, J. Phys. A 25 (1992) 5963-5976.

[Lu] J. H. Lu: Hopf algebroids and quantum gouppoids, Int. J. Math., 7 (1996) 47-70.

[NAR] Z. Nagy, J. Avan, and G. Rollet: Construction of dynamical quadratic algebras, arXiv:math.QA/0307026. 
[RS] N. Reshetikhin and M. Semenov-Tian-Shansky: Quantum R-matrices and factorization problem, J. Geom. Phys. 5 (1988), 533-550.

[S] O. Schiffmann: On classification of dynamical r-matrices. Math. Res. Lett. 5 (1998) $13-30$.

[Sch] P. Schauengburg: Bialgebras over noncommutative rings and a structure theorem for Hopf bimodules, App. Categorical Struct. 6 (1998) 193-222.

[Szl] K. Szlachányi: Finite quantum groupoids and inclusions of finite type, Mathematical physics in mathematics and physics (Sienna, 2000) 393-407, in Fields Inst. Commun. 30, Amer. Math. Soc., Providence, RI, 2001.

[Xu1] P. Xu: Quantum groupoids, Commun. Math. Phys., 216 (2001) 539-581.

[Xu2] P. Xu: Quantum Dynamical Yang-Baxter Equation Over a Nonabelian basis, Commun. Math. Phys. 226 (2002) 475-495. 